\title{
Proactive integrated handoff management in cognitive radio mobile ad hoc networks
}

\author{
Samad Nejatian ${ }^{1 *}$, Sharifah Kamilah Syed-Yusof ${ }^{1}$, Nurul Mu'azzah Abdul Latiff', Vahid Asadpour ${ }^{2}$ \\ and Haleh Hosseini ${ }^{1}$
}

\begin{abstract}
In cognitive radio networks, the secondary users (SUs) switch the data transmission to another empty spectrum band to give priority to primary users (PUs). In this paper, channel switching in cognitive radio mobile ad hoc networks (CR-MANETs) through an established route is modeled. The probability of channel availability in this route is calculated based on the PU's activity, SU's mobility, and channel heterogeneity. Based on the proposed model, the channel and link availability time are predicted. These predictions are used for channel assignment in the proposed channel allocation scheme. A handoff threshold as well as a life time threshold is used in order to reduce the handoff delay and the number of channel handoffs originating from the short channel usage time. When the channel handoff cannot be done due to the SU's mobility, the local flow handoff is performed to find an appropriate node in the vicinity of a potential link breakage and transfer the current data flow to it. The local flow handoff is performed to avoid possible flow disruption and also to reduce the delay caused by the link breakage. The study reveals that the channel heterogeneity and SU's mobility must be considered as important factors, which affect the performance of the handoff management in the CR-MANETs. The results emphasize on the improvement of the route maintenance probability after using the local flow handoff. It is also stated that the amounts of handoff requirement and handoff delay are decreased after using the predicted channel usage life time and handoff threshold time.
\end{abstract}

Keywords: Cognitive radio; Mobile ad hoc networks; Spectrum handoff; Spectrum management; Spectrum mobility

\section{Introduction}

Over the last few decades, the world has experienced an explosion of the wireless devices. This has led to some spectrum bands being heavily used, especially unlicensed bands, such as ISM bands, which can be attributed to interference and poor network performance. On the other hand, recent studies have shown that a large amount of the licensed spectrum bands remain underutilized and inefficient [1]. Hence, it was concluded that the traditional fixed spectrum allocation approach cannot efficiently regulate the spectrum access any longer. Dynamic spectrum access is an approach that permits wireless devices to use the idle frequency bands, namely spectrum holes, [1] with enabling technology of cognitive radio $(\mathrm{CR})$. CR changes its transmission parameters

\footnotetext{
* Correspondence: nsamad2@live.utm.my

${ }^{1}$ Faculty of Electrical Engineering, University Technology Malaysia, Skudai 81300, Malaysia

Full list of author information is available at the end of the article
}

based on the interaction with the surrounding environment and allows the secondary user (SU) to share the spectrum when the primary users (PUs) do not use these spectrum bands [2].

Spectrum hole availability is random due to the random appearance of PUs as well as the unpredictability of SUs' demand. The spectrum holes may shift over time and over space. In a CR system, the shifting of spectrum holes can be defined as spectrum mobility, which is cohesive to spectrum handoff. Spectrum handoff refers to the transfer of an ongoing data transmission of a CR user to another available spectrum band. Spectrum handoff is extremely challenging in CR networks, especially in cognitive radio mobile ad hoc networks (CR-MANETs), because of frequent topologic variations, limited power, limited channel transmission range, bandwidth constraints, and lack of the central controlling entity. The spectrum sharing in heterogeneous networks was proposed in [3], which considers the infrastructure- 
based network. In heterogeneous CR networks, a channel may be available over vast, mutually exclusive spectrum bands that present remarkable heterogeneity in terms of channel transmission range and channel error rate. The channels located in higher frequency bands have lower transmission ranges.

When considering the influence of node mobility on the channel availability, the effect of channel heterogeneity becomes more significant. In a CR system, node mobility and channel heterogeneity lead to frequent spectrum handoff. Thus, it is needed to propose a proper algorithm for unified, proactive integrated handoff management (PIHM) in CR-MANETs. The algorithm reduces the probability of handoff blocking, reduces route failure due to user mobility and channel heterogeneity, and maintains end-to-end route connectivity. It must also decrease the number of handoffs in the network considering the effects of different mobility events.

There are some challenges which make the PIHM difficult. The first challenge is differentiating the SU's mobility and spectrum mobility. Channel quality degradation happens because of the SU's mobility and channel heterogeneity in terms of transmission range. Therefore, there are different route failure types that necessitate different route recovery strategies. The second challenge related to integrated handoff management is finding the best new route and a channel to maintain the route while reducing the switching time and the number of handoffs. In this paper, the channel availability time is used as the main metric for channel allocation. This parameter is estimated by considering the channel heterogeneity and the SU's mobility. The handoff threshold is used to initiate a handoff procedure proactively. In some cases, the distance between two communicating nodes in a hop belonging to an active route exceeds a given threshold, and the channel handoff is not efficient because of the limited channel transmission range. In these cases, the node with the link breakage transfers the routing information to another appropriate sponsor node. This mechanism is called as local flow handoff, which aims to find a feasible route upon link breakage and transfer the traffic to another stable route based on the prediction. The motivation of this mechanism is to maintain end-to-end connectivity once a route is established for the purpose of sending data.

The rest of this paper is organized as follows. Section 2 describes the related works for spectrum handoff management. Section 3 introduces the proposed PIHM scheme, its requirements, and the design concepts. In Section 4, we propose a unified modeling and characterization of channel availability in CR-MANETs. Section 5 explains the different handoff types in CRMANETs and introduces the proposed PIHM algorithm. In Section 6, the implementation of PIHM algorithm is performed. In Section 7, the results and discussion are elaborated. Finally, Section 8 concludes the paper and presents the further suggestions.

\section{Related works}

There are a few studies related to spectrum handoff in CR-MANETs. Giupponi and Perez-Neira [4] proposed a fuzzy-based spectrum handoff decision-making approach employing two fuzzy logic controllers. Each SU estimates the distances between itself and all the active PUs in the surrounding area using the first fuzzy logic controller. The other fuzzy logic controller determines whether the SU has to do a spectrum handoff or not. In some cases, the SU can avoid performing a spectrum handoff by appropriate adjustment of its transmission power. Feng et al. [5] developed a spectrum handoff technique from a single link concept to a multilink spectrum handoff scheme. The proposed algorithm tries to minimize the total link cost by taking into account the end-to-end network connectivity constraint. Another major contribution of this paper was that the rerouting mechanism was performed before the spectrum handoff event to increase the system throughput.

Song and Xie [6,7] proposed a proactive spectrum handoff configuration based on the statistics of observed channel utilization. The network coordination and rendezvous issues were solved in this spectrum handoff scheme without using a common control channel. The collision among SUs was also deleted by a distributed channel determination scheme. Damljanovic explained the proper solutions and spectrum mobility necessities in cognitive radio networks [8]. Duan and Li proposed a spectrum handoff strategy in which the optimal spectrum band was chosen based on a multiplex criterion considering the estimated transmission time, the PU presence probability, and the spectrum availability time [9]. A cooperative spectrum sensing scheme was used to predict the spectrum idleness. The authors use a geolocation method in order to consider a spectrum handoff in the space domain. The simulation results indicate that the proposed spectrum handoff scheme outperforms conventional methods in terms of spectrum handoff delay. In this paper, the authors considered a per hop basis scheme. Moreover, they did not consider all the effective parameters on the spectrum handoff such as channel heterogeneity. Wu and Harms [10] proposed a proactive flow handoff for legacy mobile ad hoc networks. The major contribution of this scheme was maintaining end-to-end connectivity after a flow was established. This scheme introduced the consideration of user mobility and location information. Abhilash et al. [11] proposed a preemptive route maintenance scheme in which an established route is repaired before it breaks by considering the mobile ad hoc user's location 
information. They called this scheme local router handoff and implemented it into the Ad hoc On-demand Distance Vector protocol (AODV). Based on the results, the throughput of the system was increased under certain conditions. Caleffi et al. [12] proposed an optimal routing metric for cognitive radio ad hoc networks that considers the route diversity effects to overcome unoptimality and un-accuracy in CR routing. In this paper, the route diversity provided by the intermediate nodes is considered to calculate the end-to-end delay of a route considering the unique characteristics of cognitive radio networks. The results of the analytical model and simulation reveal the benefits of the proposed routing metric for cognitive radio ad hoc networks. In the proposed model, the authors did not consider the effects of the SU's mobility and spectrum heterogeneity on the routing metric. Chehata et al. [13] introduced the CR-AODV as a multi-radio multi-channel on-demand scheme that can manage the data transmission of cognitive users. Cacciapuoti et al. [14] proposed a reactive routing protocol by evaluating the feasibility of reactive routing for CR-MANETs. Nejatian et al. [15] pioneered handoff management in CR-MANETs. Factors and types of mobility were mentioned, which necessitate integrated mobility and handoff management in CR-MANETs. In this paper, a conceptual model was proposed for integrated handoff management in CR-MANETs.

Maintaining the optimal routes in CR-MANETs is extremely difficult because of the randomness PU's activity, SU's mobility, and channel quality [16]. Hence, a framework must be introduced for integrated handoff management and local flow handoff in CR-MANETs. Based on a review of the literature, there is no study that considers proactive unified spectrum handoff in CRMANETs. In the next section, the proposed PIHM framework is explained, and assumptions regarding the network architecture are introduced.

\section{Proposed PIHM framework}

Figure 1 shows the framework for integrated handoff management in CR-MANETs. The proposed scheme adapts to unpredictable events and makes a decision on the SU's data transmission without causing any interference to the PUs, while maintaining the end-to-end connectivity. The proposed PIHM scheme is equipped with an algorithm to identify appropriate spectrum bands based on the channel qualities, spectrum, and node mobility. The spectrum analysis part needs information about the situations and the mobility of the SUs. Hence, a precise and cooperative environment and location-aware mechanism is necessary. In a precise spectrum handoff decision making, the link and channel availability time must be considered. The decision-making procedure estimates the channel availability time based on the SUs' mobility and the channel transmission range.

\section{Characterization of channel availability in CR-MANETs}

To design the PIHM protocol for a CR-MANET, the PU's activity, SU's mobility, channel quality degradation,

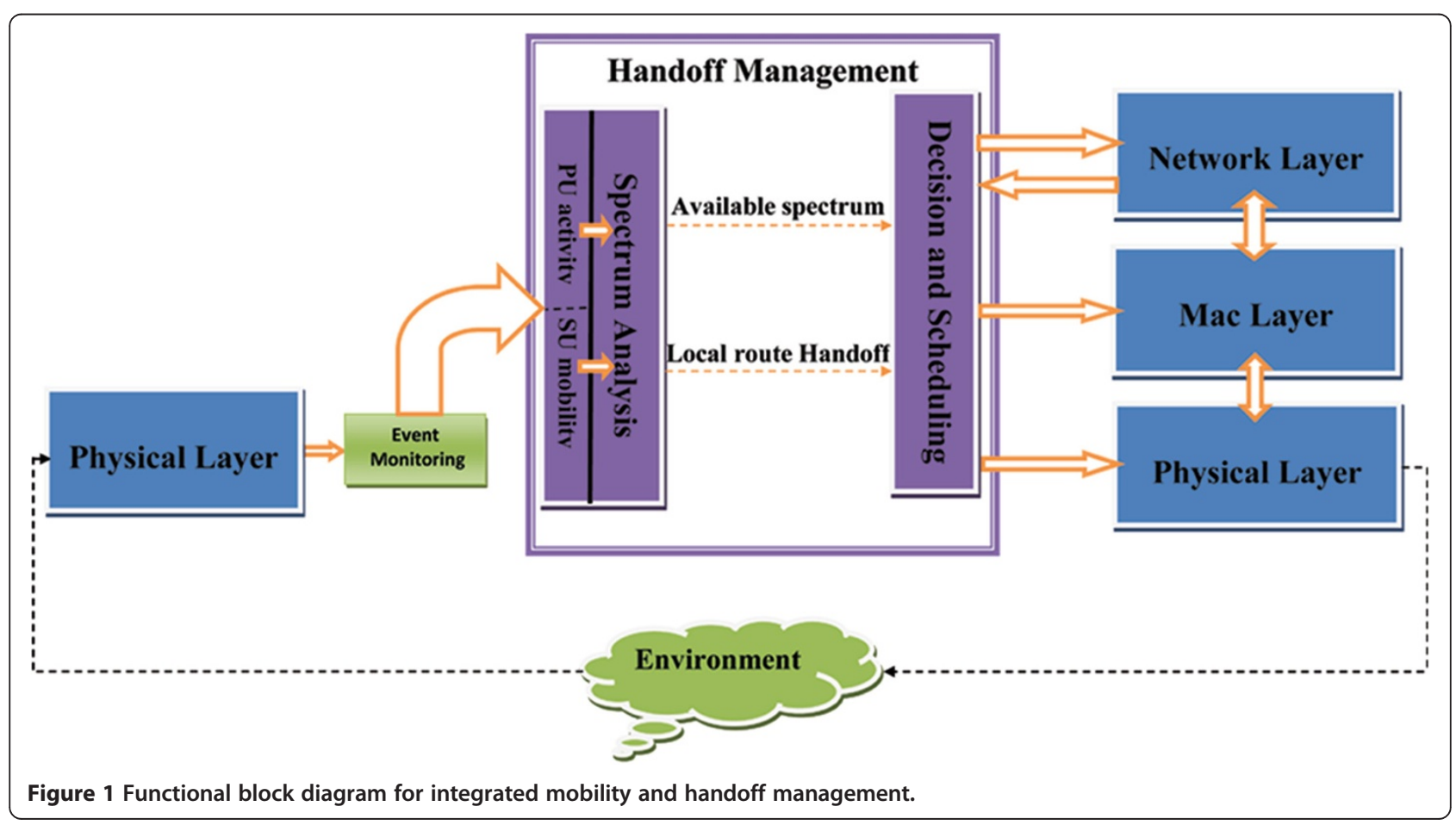


and topologic variations are considered jointly. Herein, the probability of channel availability in a CR-MANET is calculated as a unified formula considering the effects of all the above mentioned events, number of nodes, and node and channel transmission range as well as the number of channels in the network. This integrated equation for channel availability in CR-MANETs is useful for better understanding of the PIHM concept in the CR-MANETs. Table 1 shows the symbols used in this paper and their definitions.

\subsection{System description}

Throughout this paper, it is assumed that the SU is equipped with multiradio, multichannel, and common control channel signaling features. Suppose that there are $L$ different channel types in a heterogeneous PU network. The maximum number of channels that can be accessed by the SU at a time is $C$. The $C$ channels can be defined by the set of $\mathrm{T}$, which belong to the PU network. These channels are classified into $L$ types according to their different transmission ranges that the cardinality of $|\mathrm{T}|=\mathrm{L}$ (see Figure 2). Channels with different transmission ranges belong to a different set of spectrum pools. The set of each type can be shown by $T_{l}$ in which $l \in \mathbf{S}, \mathbf{S}=\{1,2, \ldots, L\},\left|T_{l}\right|=C_{l}, \mathbf{T}=\left\{T_{1}, \ldots\right.$, $\left.T_{L}\right\}$, and $C=C_{1}+\ldots+C_{L}$. Depending on the PU activities, any SU can access up to $C$ channels at any position. The number of detected channels of type $l$ by a special node is $c_{l}$, and the total number of detected channels at a node is $c=c_{1}+c_{2}+\ldots+c_{L}$. The transmission range of channels of type $T_{l}$ is $R_{l}$. Given the channel transmission range classification, we can model the channel heterogeneity. Assume a pair of SU transmitter-receivers, which transmit and receive, respectively, using channel of type $T_{l}$ for communication, with a distance between them of less than $R_{l}$. When the SUs move and their distance exceeds $R_{l}$, the transmitting node must change and choose another channel. In this case, the required channel must have a transmission range longer than $R_{l}$ (Figure 3). There are also $L$ types of PU; each of them can work only on a channel of type $l$. Once a PU of type $l$ becomes active and there is no empty channel of type $l$, in the case which a SU has occupied a channel of type $l$, the SU must vacate the channel and submit it to the PU.

\subsection{Characterization of channel availability and spectrum mobility in CR-MANETs}

Consider an established route from the source node $\mathrm{S}$ to the destination node D (Figure 4). We define the parameter $p$ as the probability of single channel availability at a node by considering the PU activity, and $P_{\text {cat }}$ is the probability of single channel availability per hop between two nodes. Because the channel availability on all nodes is independent of each other, we can consider different
Table 1 Symbols used in this paper and their definitions

\begin{tabular}{|c|c|}
\hline Symbol & Meaning \\
\hline$R_{\mathrm{T}}$ & Node transmission range \\
\hline C & Total number of available channels \\
\hline$c$ & Number of possible channels at each node \\
\hline 1 & Channel type \\
\hline L & Total number of channel types \\
\hline$c_{1}$ & $\begin{array}{l}\text { Number of detected channels of each type at } \\
\text { each node index }\end{array}$ \\
\hline$p$ & Probability of a particular channel availability at each node \\
\hline$P_{\text {cat }, T_{i}}$ & $\begin{array}{l}\text { Probability that there is at least one single channel among } \\
\text { c channels between two nodes }\end{array}$ \\
\hline$P_{c a r, c}$ & $\begin{array}{l}\text { Probability that there is at least one common channel } \\
\text { among all hops in a route }\end{array}$ \\
\hline$\lambda$ & Poisson density of nodes' spatial distribution in the network \\
\hline$R_{l}$ & Transmission range of a channel of type / \\
\hline$P_{\text {snd }}$ & Signal power at transmitting antenna \\
\hline$P_{r}$ & Receiving power at distance $r$ \\
\hline$P_{\text {STPCH }}$ & Signal power threshold for preemptive channel handoff \\
\hline 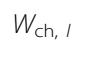 & $\begin{array}{l}\text { Warning distance for nodes communicating on a channel } \\
\text { of type / }\end{array}$ \\
\hline$W_{\text {link }}$ & Warning distance for preemptive local flow handoff region \\
\hline$V_{\text {relative }}$ & Relative speed \\
\hline$P_{\text {CTRII }}$ & Received power at channel transmission range of type I \\
\hline $\mathrm{SHTH}, \mathrm{I}$ & Handoff threshold for the preemptive channel handoff \\
\hline LHTH & Handoff threshold for the preemptive local flow handoff \\
\hline$P_{\text {SLFTH }}$ & Signal power threshold for preemptive flow handoff \\
\hline$P_{\text {NTR }}$ & Power received at the node transmission range, $R_{l}$ \\
\hline$t_{\mathrm{w}}$ & Interval from the warning till the break \\
\hline$t_{\text {ava }, T_{1}}$ & Channel availability time for the channel of type I \\
\hline$t_{\text {ava }, T_{i}, H_{i}}$ & $\begin{array}{l}\text { Channel availability time for the channel of type / available } \\
\text { in hop } i\end{array}$ \\
\hline$t_{a v a, L}$ & Link availability time \\
\hline$W_{T,}, D_{i-1, i}$ & Location weight for case $D_{i-1} i$ and channel of type $T_{l}$ \\
\hline$h_{i, l}$ & $\begin{array}{l}\text { Possibility of using the channel of type } I \text {, detected by both } \\
\text { nodes belonging to the hop } i\left(H_{i}\right)\end{array}$ \\
\hline$\eta_{H_{i}}, c_{1 z}$ & $\begin{array}{l}\text { Possibility of using the channel } z \text { of type / for communicating } \\
\text { in hop } i\end{array}$ \\
\hline$\xi_{H_{i}, T_{i}}$ & $\begin{array}{l}\text { Possibility of using the channel of type / for data transmission } \\
\text { in hop } i\end{array}$ \\
\hline$A M_{C_{12}}$ & Channel allocation metric for channel $z$ of type I \\
\hline $\mathrm{C}_{H_{i}}$ & $\begin{array}{l}\text { The set of detected channels by the two nodes belonging } \\
\text { to the hop } i\end{array}$ \\
\hline$C_{H_{i, j}}$ & $\begin{array}{l}\text { Channel } z \text { of type / belonging to the set of detected channels } \\
\text { by the two nodes belonging to the hop } i\end{array}$ \\
\hline $\mathrm{C}_{H_{j}}$,usable & $\begin{array}{l}\text { The set detected channel that can be used for } \\
\text { communication in hop } i\end{array}$ \\
\hline
\end{tabular}

hops separately to estimate the probability of channel availability through the established route. Thus, $P_{\text {cat }}$ is as follows: 


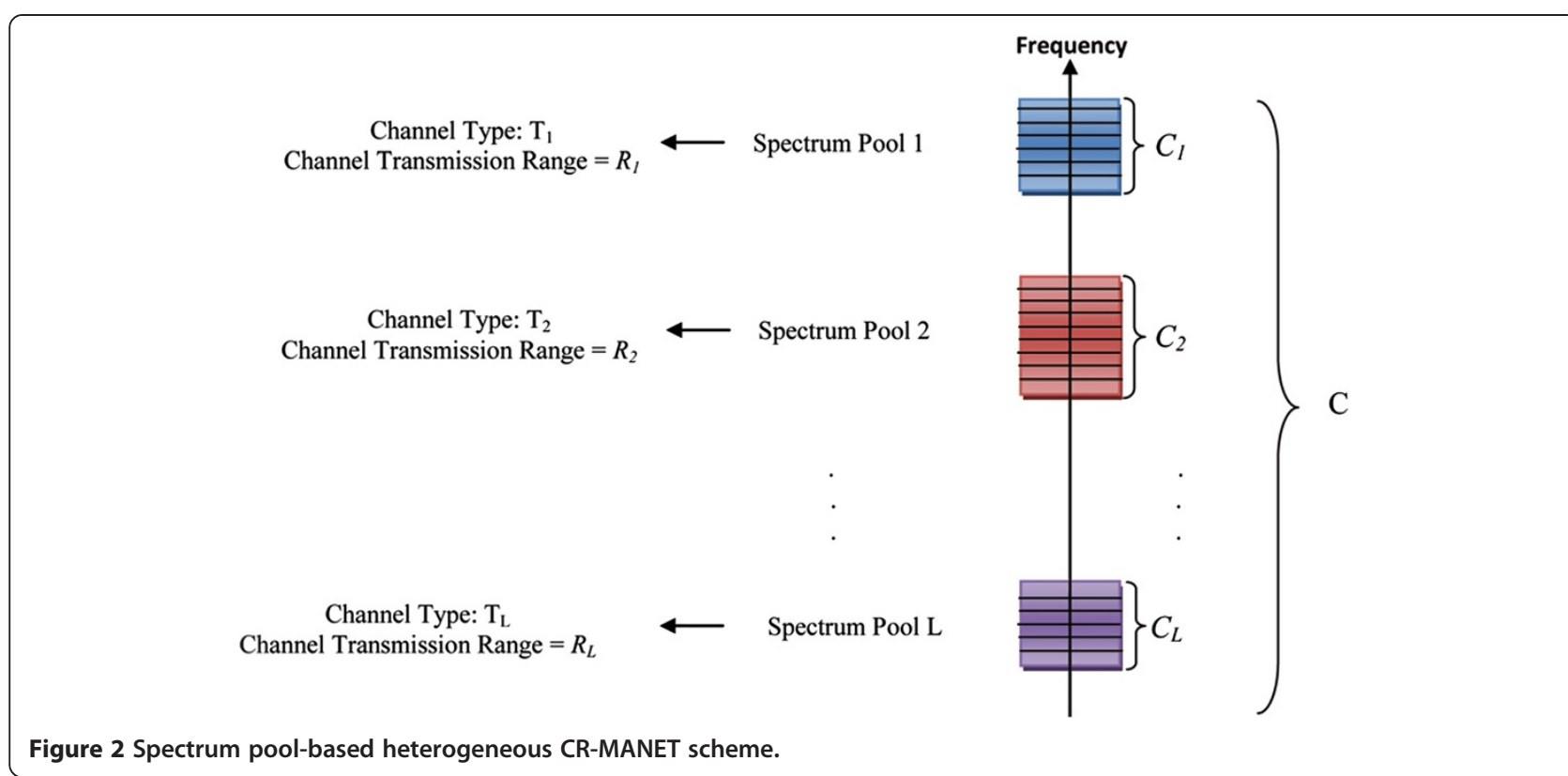

$$
P_{c a t}=p^{2}
$$

Consequently,

$$
\bar{P}_{c a t}=1-p^{2}
$$

The parameter $\bar{P}_{c a t, c}$ is defined as the probability that there is no single channel among $c$ channels between two nodes, which is defined as follows:

$$
\bar{P}_{c a t, c}=\left(\bar{P}_{c a t}\right)^{c}=\left(1-p^{2}\right)^{c}
$$

According to (3), the probability of existence of at least one single channel among $c$ channels in a hop is equal to

$$
P_{c a t, c}=1-\bar{P}_{c a t, c}=1-\left(1-p^{2}\right)^{c}
$$

Suppose that the total number of nodes in a route is equal to $n$. Thus, we have $n-1$ hops. Based on (4) and

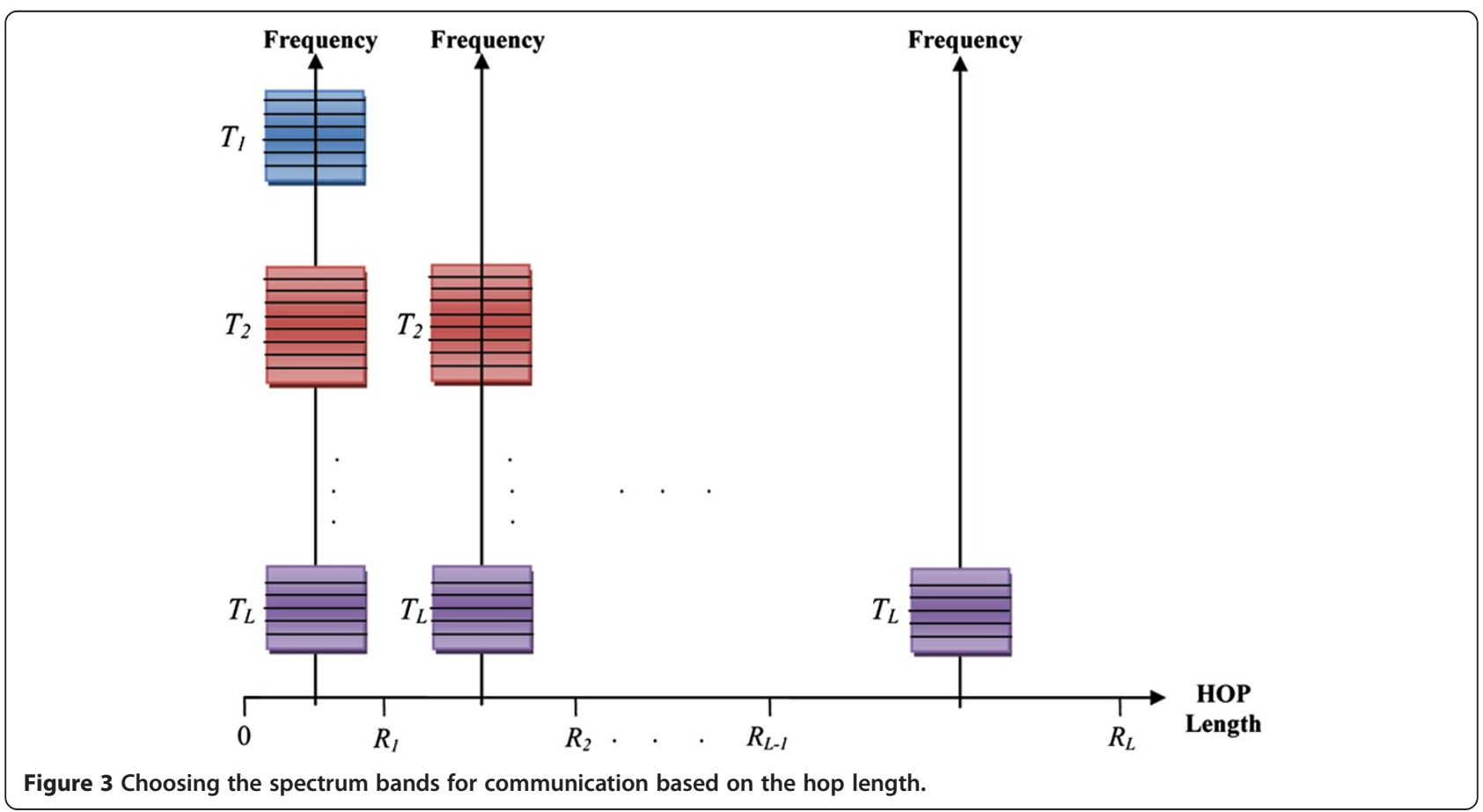




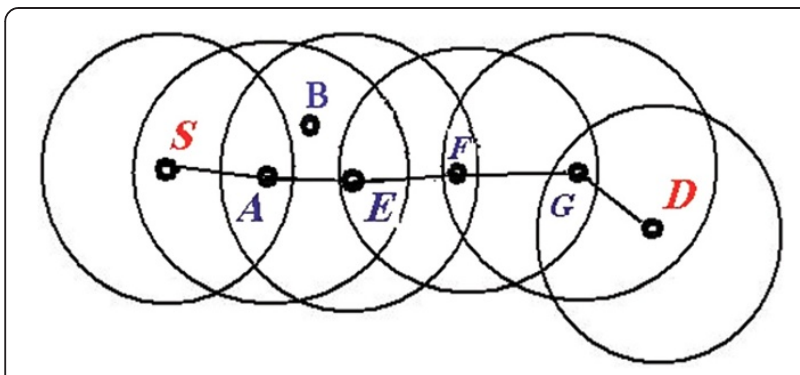

Figure 4 An established route from a source node to a destination node.

the existence of $n-1$ hops through a route, we define the $P_{\mathrm{car}, c}$ as the probability of the existence of at least one channel among all hops in a route, which is equal to

$$
P_{c a r, c}=\left(P_{c a t, c}\right)^{n-1}=\left(1-\left(1-p^{2}\right)^{c}\right)^{n-1}
$$

The effects of the spectrum heterogeneity and mobility of the SU on the probability of channel availability must also be considered. In a heterogeneous network, each channel experiences various levels of packet error rate and different channel transmission ranges. In the initial condition when the nodes are assumed to be fixed, different channels will have different transmission ranges. A channel with a lower frequency range needs lower transmission power. Thus, in a heterogeneous network with different channel transmission ranges, the distance between the SUs affects the probability of channel availability.

We define $\bar{P}_{c a t, T_{l}}$ as the probability of unavailability of a single channel of type $l$ among $c_{l}$ channels between two nodes as follows:

$$
\bar{P}_{c a t, T_{l}}=\left(\bar{P}_{c a t}\right)^{c_{l}}=\left(1-p^{2}\right)^{c_{l}}
$$

Based on (6), the probability that there is at least one single channel of type $T_{l}$ among $c_{l}$ channels between two nodes is

$$
P_{c a t, T_{l}}=1 \bar{P}_{c a t, T_{l}}=1-\left(1-p^{2}\right)^{c_{l}}
$$

Suppose that $n$ is the total number of nodes in a route, which means that there are $n-1$ hops in the route. The probability of channel availability within a hop depends on the distance between nodes, $R_{l}$. Given that there are $r_{1}$ hops in the route with distance $d$ where $R_{0}<d<R_{1}$, there are $r_{2}$ hops in the route with distance $d$ where $R_{1}<d<R_{2}, \ldots$, and there are $r L$ hops in the route with distance $d$ where $R_{L-1}<d<R_{L}$, such that $r_{1}+r_{2}+\ldots+$ $r_{L}=n-1$. Considering (7) and the law of the total probability [17], the $P_{\mathrm{car}, c}$ is defined as the probability of existence of at least one common channel among all hops in a route as follows:

$$
\begin{aligned}
P_{c a r, c}= & P\left(r_{1}\right)\left[1-\left(1-p^{2}\right)^{c}\right]^{r_{1}}+P\left(r_{2}\right)\left[1-\left(1-p^{2}\right)^{c-c_{1}}\right]^{r_{2}} \\
& +\ldots+P\left(r_{L-1}\right)\left[1-\left(1-p^{2}\right)^{c-c_{1}-\ldots-c_{l-2}}\right]^{r_{L-1}} \\
& +P\left(r_{l}\right)\left[1-\left(1-p^{2}\right)^{c_{L}}\right]^{r_{L}} \\
= & \sum_{i=1}^{L} P\left(r_{i}\right)\left[1-\left(1-p^{2}\right)^{c-} \sum_{j=0}^{i-1} c_{j}\right]^{r_{i}} \quad \mid c_{0}=0
\end{aligned}
$$

where $P\left(r_{i}\right)$ is the probability of existence of the number of $r_{i}$ hops in the route, with distance $d$, where $R_{i-1}<d<R_{i}$. We define the $P\left(R_{i}\right)$ as the probability that the length of the hop between two adjacent nodes in the route is less than $R_{i}$ and longer than $R_{i-1}$. Because the distances between adjacent nodes in a route are independent random variables, we have [18]

$$
P\left(r_{i}\right)=\left(P\left(R_{i}\right)\right)^{r_{i}}
$$

Consequently, based on (8), (9), and $\sum_{i=1}^{L} r_{i}=n-1$, we have

$$
P_{\mathrm{car}, c}=\left[\sum_{i=1}^{L} P\left(R_{i}\right)\left[1-\left(1-p^{2}\right)^{c-} \sum_{j=0}^{i-1} c_{j}\right]\right]^{n-1} \text { and } c_{0}=0
$$

Because $p$ is the probability of single channel availability at a node given the activity of the PU, different traffic models for the activity of the PU can be applied. Here, an alternating renewal two state birthdeath process with a death rate $\alpha$ and a birth rate $\beta$ is considered to model the activity of the PU. The arrival time of each user is independent, so the transmission time of each user is considered as a Poisson arrival process. Therefore, the length of off and on periods has an exponential distribution with means of $1 / \alpha$ and $1 / \beta$, respectively. Consequently, the activity of the PU can be stated as follows:

$$
P_{\text {on }}=\frac{\beta}{\alpha+\beta} \text { and } P_{\text {off }}=\frac{\alpha}{\alpha+\beta}
$$

When the PU is idle, the respective channel is available for SU transmission. Thus, the probability of single channel availability at a node is equal to $P_{\text {off }}$, and it can be stated that 


$$
p=\frac{\alpha}{\alpha+\beta}
$$

Substituting (12) in (8), we have

$$
P_{c a r, c}=\sum_{i=1}^{L} P\left(r_{i}\right)\left[1-\left(1-\left(\frac{\alpha}{\alpha+\beta}\right)^{2}\right)^{c-} \sum_{j=0}^{i-1} c_{j}\right]^{r_{i}} \text { in which } c_{0}=0
$$

In some cases, the activities of the PUs on different channels are unequal. Based on the superposition property, multiple Poisson processes with different death rates $\alpha_{k}$ and birth rates $\beta_{k}$ can be merged and considered as a single alternating renewal two state birth-death process, with a death rate $\alpha_{k}$ and a birth rate $\beta_{k}$. Therefore, a single PU can be modeled in each communication channel. Thus, we can assume

$$
p=\frac{\alpha_{k}}{\alpha_{k}+\beta_{k}}
$$

Therefore, (8) changes to (15) as follows:

$$
\begin{aligned}
P_{c a r, c} & =\sum_{i=1}^{L} P\left(r_{i}\right)\left[1-\prod_{k=1}^{c-} \sum_{j=0}^{i-1} c_{j}\left(1-\left(\frac{\alpha_{k}}{\alpha_{k}+\beta_{k}}\right)^{2}\right)\right]^{r_{i}} \\
& =\left[\sum_{i=1}^{L} P\left(R_{i}\right)\left(1-\prod_{k=1}^{c-\sum_{j=0}^{i-1} c_{j}}\left(1-\left(\frac{\alpha_{k}}{\alpha_{k}+\beta_{k}}\right)^{2}\right)\right)\right]^{n-1}
\end{aligned}
$$

In practical CR networks, the sensing capability, which influences the reliable probability of channel availability, is not perfect. However, in this work, we follow the assumption of perfect sensing in which the probabilities of both false alarms and miss detections are zero.

Let the node transmission power be fixed to $R_{T}$. In fact, the transmitting range with a similar transmitting power on wireless spectrum bands with different frequencies is unequal. We assume that $R_{1}<\ldots .<R_{\mathrm{L}}<R_{\mathrm{T}}$. The probability of $P\left(R_{i}\right)$ is dependent on the number of hops and also the limit of the transmission range. To demonstrate the effects of channels with different transmission ranges on the system, we model the spatial distribution of mobile nodes as a Poisson variable with density $\lambda$. Thus, [19]

$$
\begin{aligned}
& \operatorname{Pr}(\text { There are } k \text { nodes in a region with disc area } S) \\
& =\frac{\lambda S^{k} \exp (-\lambda S)}{k !}, k=0,1,2,3, \ldots
\end{aligned}
$$

Without the loss of generality, this can be considered as a snapshot of the mobile radio network, by which we can calculate $P\left(R_{i}\right)$. The number of neighboring nodes in a disc area with radius $R_{T}$ is equal to

$$
N=\lambda \pi R_{T}^{2}
$$

The probability that there is no neighbor node in the forward direction in the disc with radius $R_{T}$ is also expressed as $\exp \left(-{ }^{N} / 2\right)$; therefore,

$$
\left.P\right|_{\text {finding a node in a disc with radius } R_{T}}=1-\exp \left({ }^{-} N / 2\right)
$$

Assume that the random variable $d$ denotes the distance between a pair of transmitter and receiver nodes and the $f_{d}\left(R_{i}\right)$ represents its probability density function. The probability distribution function of $d$ is as follows:

$$
F_{\mathrm{d}}\left(R_{i}\right)=\operatorname{Pr}\left(d<R_{i}\right)=\frac{1-\exp \left(-\lambda \pi R_{i}{ }^{2} / 2\right)}{1-e^{-\frac{N}{2}}} \text {, in which } 0<R_{i}<R_{T} .
$$

Thus, we can conclude that

$$
\begin{aligned}
\operatorname{Pr}\left(R_{i-1}<d<R_{i}\right)= & F_{d}\left(R_{i}\right)-F_{d}\left(R_{i-1}\right) \\
= & \frac{1-\exp \left(-\lambda \pi R_{i}^{2} / 2\right)}{\frac{N}{2}}-\frac{1-\exp \left(-\lambda \pi R_{i-1}^{2} / 2\right)}{1-e^{-\frac{N}{2}}} \\
= & \frac{\exp \left(-\lambda \pi R_{i-1}^{2} / 2\right)-\exp \left(-\lambda \pi R_{i}^{2} / 2\right)}{1-e^{-\frac{N}{2}}} \\
& \text { in which } \quad 0<R_{i-1}<R_{i}<R_{T} \mid R_{0}=0
\end{aligned}
$$

Finally, we have

$$
\begin{aligned}
P_{c a r, c}=\left(\sum_{i=1}^{L} \frac{\exp \left(-\lambda \pi R_{i-1}^{2} / 2\right)-\exp \left(-\lambda \pi R_{i}^{2} / 2\right)}{1-e^{-\frac{N}{2}}}\right. \\
\left.\quad \times\left(1-\prod_{k=1}^{c-\sum_{j=0}^{i-1} c_{j}}\left(1-\left(\frac{\alpha_{k}}{\alpha_{k}+\beta_{k}}\right) 2\right)\right)\right)^{n-1}
\end{aligned}
$$

In the case of a network with homogenous channels, all of the channels have the same transmission range and belong to the same type. If we consider the node transmission range, for example, as $R_{T}=R_{1}$, then all of the channels belong to type 1 . Thus, we have

$$
\left.\sum_{j=o}^{i-1} c_{j}\right|_{i \neq 1}=0 \quad \text { and } \quad \sum_{i=1}^{L} r_{i}=r_{1}=n-1
$$

Therefore, (21) reduces to (5) in which the system provides homogeneous channels.

Based on the channel availability modeling, the unified spectrum handoff scheme must be proposed to include 
different mobility events in CR-MANETs, such as spectrum and user mobility, channel quality degradation, and topologic variation.

\section{Proposed PIHM algorithm}

To propose the PIHM algorithm, the different scenarios that cause handoff initiation through an established route in a CR-MANET are introduced. In CR-MANETs, the available frequency bands change over time. On the other hand, during the movement of an intermediate node, the route may be broken and it should be rectified. The local flow handoff technique is useful to avoid route breaking. As shown in Figure 5, a route starting from the source node $\mathrm{S}$ to the destination node $\mathrm{D}$ has been established. Three various scenarios can initiate a handoff in this route. These scenarios can be defined as follows:

\subsection{The SU's mobility}

As shown in Figure 5a, route failure occurs when either node $\mathrm{B}$ or node $\mathrm{F}$ moves such that any channel cannot support their communication. Before the route failure, a local flow handoff is performed. To perform local flow handoff, a certain amount of overlapping of transmission range between node $\mathrm{A}$, node $\mathrm{F}$ and the intermediate node that will take responsibility for routing the packets is needed.

\subsection{The PU's activity dominates}

Figure 5b shows the second scenario when the PU's activity in a neighboring area of node $\mathrm{C}$ may cause the links A-E or E-F to fail. This route failure occurs once the PU starts its communication or node $\mathrm{C}$ is mobile and enters the PU's activity area.

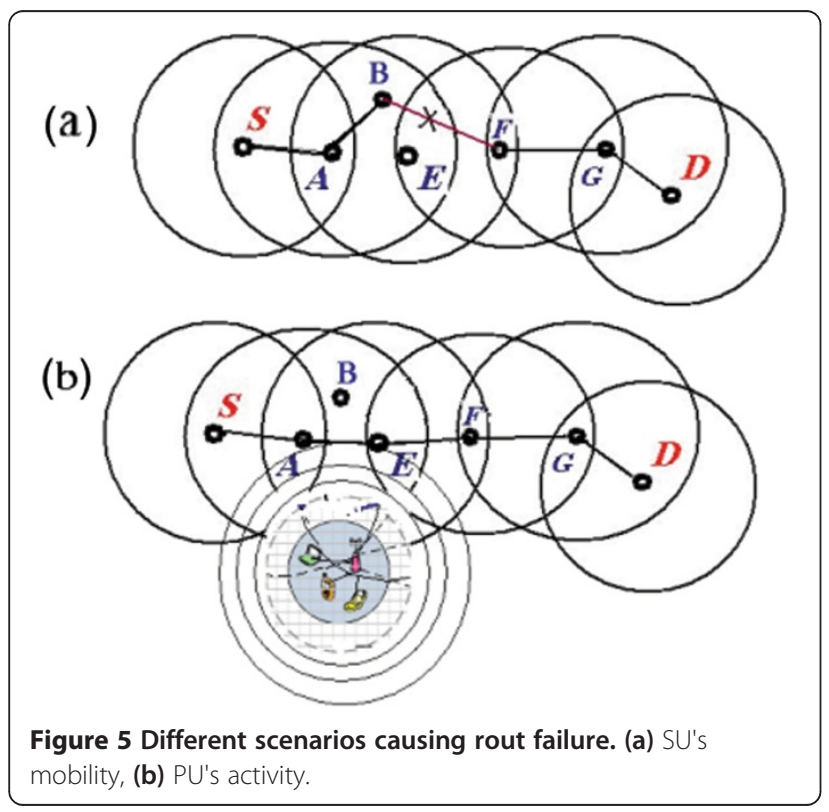

\subsection{Spectrum heterogeneity and different channel transmission range}

The mobility of a CR user can also lead to spectrum handoff due to spectrum heterogeneity and a variety channel transmission ranges. Assume that two mobile nodes with a distance less than $R_{l}$ are communicating in an active route while they are using a channel of type $l$ for their data transmission. When their distance exceeds the $R_{l}$, they must change their communication channel and find a channel with a transmission range longer than $R_{l}$.

Based on these different scenarios, the various spectrum handoff types are proposed in Figure 6. They are defined as follows:

- Forced intra-pool spectrum handoff: The operation frequency of the SU is changed to another spectrum band in the same spectrum pool. This type of handoff happens because of the appearance of the PU.

- Forced inter-pool spectrum handoff: The operation frequency of the $\mathrm{SU}$ is changed to another spectrum band in a different spectrum pool because of the appearance of the PU.

- Inter-pool spectrum handoff: The CR user changes its spectrum bands from one spectrum pool to another different spectrum pool. This type of spectrum handoff occurs because of the mobility and channel quality degradation of the SU.

- Local flow handoff: Due to the SU mobility, there is no channel that can support the data transmission.

Considering the different handoff types in CRMANETs discussed in the previous section, Figure 7 illustrates the developed algorithm for PIHM scheme in which, based on the handoff threshold, the decisionmaking unit can initiate the handoff. As will be explained in the next subsection, the decision-making unit can initiate the handoff based on the handoff threshold. When the spectrum handoff cannot be implemented in one hop, the local flow handoff will be established to maintain communication.

\section{Implementation of the proposed PIHM algorithm}

6.1 Channel and local flow handoff prediction

Based on different introduced handoff types, two different preemptive handoff regions are defined. These two regions are the preemptive channel handoff region and the preemptive local flow handoff region. As illustrated in Figure 8, the first region is determined based on the channel transmission range, which is different for various channel types. The second region is determined 


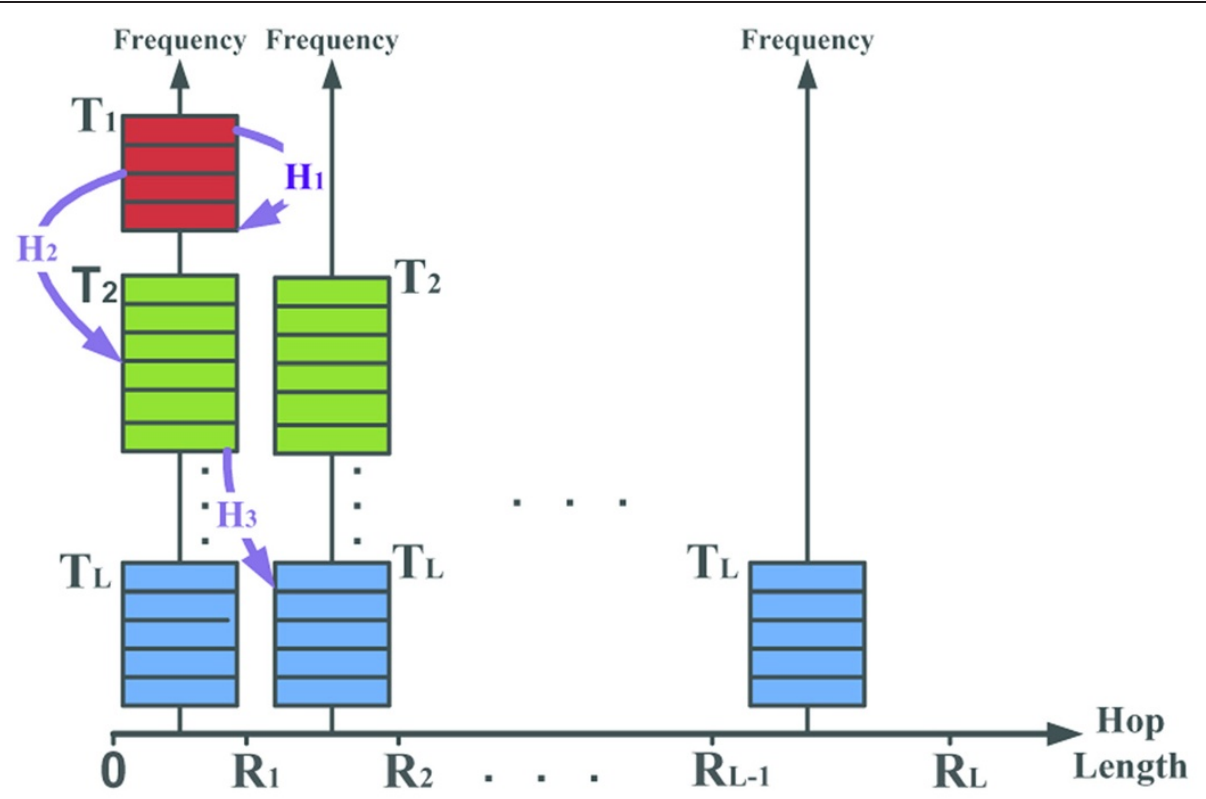

\section{H1: Forced Intra Pool Spectrum handoff $\quad H_{2}$ : Forced Inter Pool Spectrum handoff}

H3: Inter Pool Spectrum handoff

Figure 6 Different handoff types.

by the node transmission range. Because the node transmission range is constant, there is only one preemptive flow handoff region. Two different handoff thresholds are also defined related to each area. The first handoff threshold, which is related to the preemptive channel handoff region, is called the spectrum handoff threshold (SHTH). The second handoff threshold is the local flow handoff threshold (LHTH), which is related to the preemptive local flow handoff region. These two handoff thresholds are used to initiate the handoff procedure due to node mobility and channel quality degradation. Suppose that nodes B and F are communicating with each other. When either node B or node F moves such that the current channel cannot support their data transmissions, they must perform the handoff of the current channel and transfer their ongoing data transmission into another channel with a higher transmission range. If nodes $B$ and $K$ are communicating with each other, then when either node $B$ or node $K$ moves such that there is no channel to support their data transmissions, local flow handoff is performed before the link breaks.

Here, two different handoff thresholds are related to the signal power threshold. In this paper, the signal power of hello packets is used to approximate the distance between the transmitter and the receiver.

Suppose that $P_{\text {snd }}$ is the hello packet signal power at the transmitting antenna and $P_{r}$ is the receiving power at distance $r$. Based on [20], the signal power in a free space decreases with distance such that:

$$
P_{r}=\frac{P_{\text {snd }}}{r^{n}}
$$

where $n$ is a number typically between 2 and 4 (e.g., equal to 2 near the transmitter until a certain point at which $n$ becomes 4 ). Because the two preemptive regions are near the maximum transmission range, we model the drop in the signal power by $1 / r^{4}$ throughout these regions. It means that:

$$
P_{r}=\frac{P_{\text {snd }}}{r^{4}}
$$

Based on (24), the signal power threshold for preemptive channel handoff $\left(P_{\mathrm{STPCH}}\right)$ is expressed as follows:

$$
P_{S T P C H, l}=\frac{P_{\text {snd }}}{\left(R_{l}-W_{c h, l}\right)^{4}}=\frac{P_{\text {snd }}}{\left(R_{l}-\left(v_{\text {relative }} \times t_{\mathrm{w}, l}\right)\right)^{4}}
$$

where the $W_{\mathrm{ch}, l}$ is the warning distance for nodes communicating on a channel of type $l, v_{\text {relative }}$ is the relative speed of nodes, and $t_{\mathrm{w}, l}$ is the warning interval. The minimum power received by the receiver is the power 


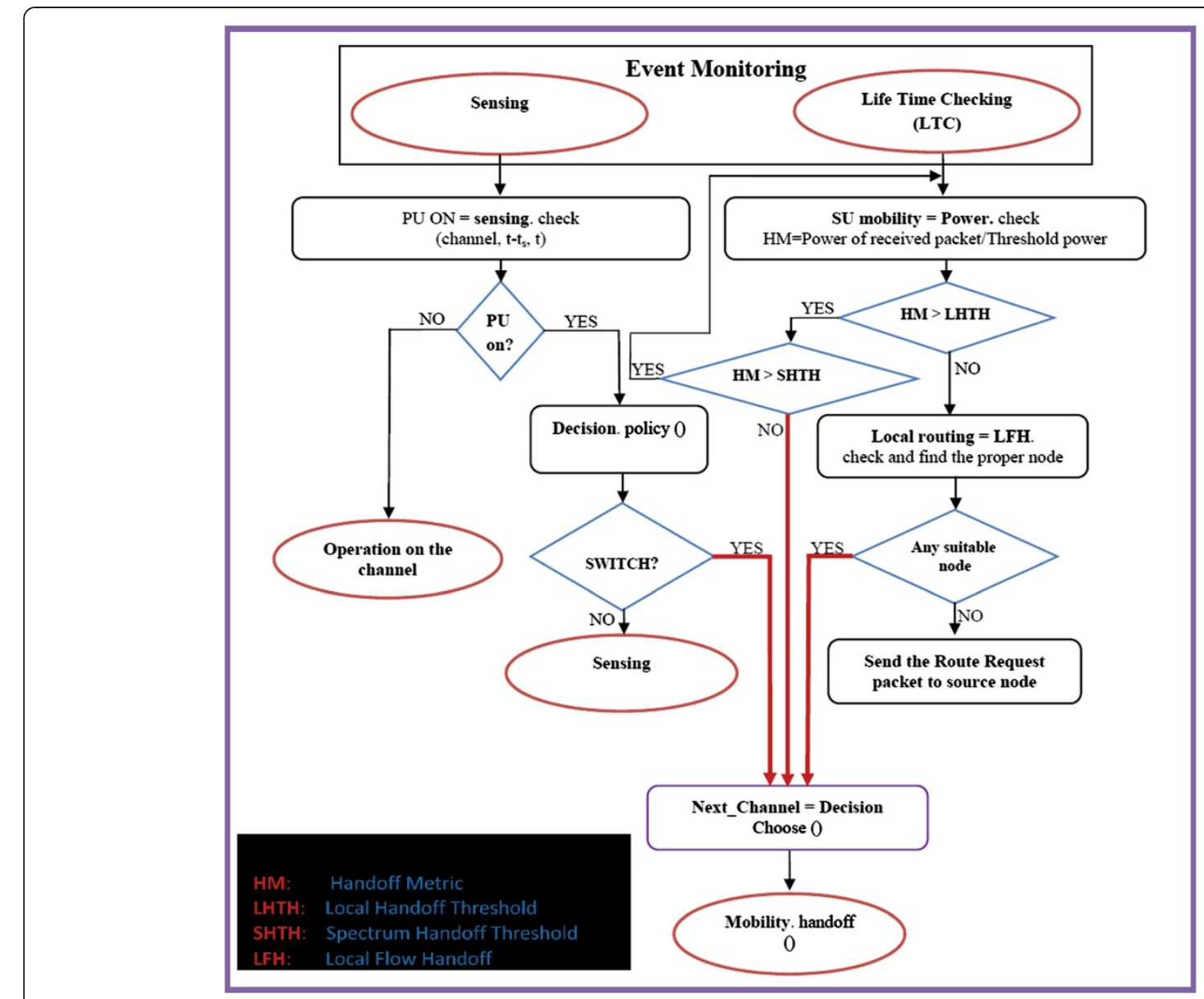

Figure 7 Integrated mobility and handoff management algorithm.

at the channel transmission range, $P_{\mathrm{CTR}, l}$ is expressed as follows:

$$
P_{C T R, l}=\frac{P_{\text {snd }}}{R_{l}^{4}}
$$

The SHTH is shown as follows:

$$
\text { SHTH }, l=\frac{P_{\text {STPCH }, l}}{P_{C T R, l}}=\frac{R_{l}{ }^{4}}{\left(R_{l}-\left(v_{\text {relative }} \times t_{\mathrm{w}, l}\right)\right)^{4}}
$$

Similarly, the handoff threshold for the preemptive LHTH is stated as follows:

$$
L H T H=\frac{P_{S L F T H}}{P_{N T R}}=\frac{R_{T}{ }^{4}}{\left(R_{T}-W_{\text {link }}\right)^{4}}
$$

where $P_{\text {SLFTH }}$ is the signal power threshold for preemptive flow handoff and $P_{\mathrm{NTR}}$ indicates the minimum power received by the receiver at the node transmission range, $R_{T}$. $W_{\text {link }}$ is the warning distance for preemptive local flow handoff region.
The $t_{\mathrm{w}}$, which is the interval from the warning till the break, needs to be greater than or equal to the necessary time for performing the handoff.

\subsection{Channel and link usage time prediction}

The concern in channel usage time prediction is about the situation that two communicating mobile nodes move out of the current channel transmission range. While, the concern in the link usage time prediction is referred to the situation in which two communicating mobile nodes move out of the node transmission range. Based on (27), to estimate the availability time, relative speed of the nodes is determined. The authors of [3-21] have employed the SU's mobility to predict the availability time for cognitive radio networks. However, they estimate the link availability time using relative movement information such as relative speed and relative direction of the communicating nodes. The proposed schemes are required to use the GPS or other technologies for getting the positions and velocities of the nodes which can increase the system expenses. Here, the relative movement 


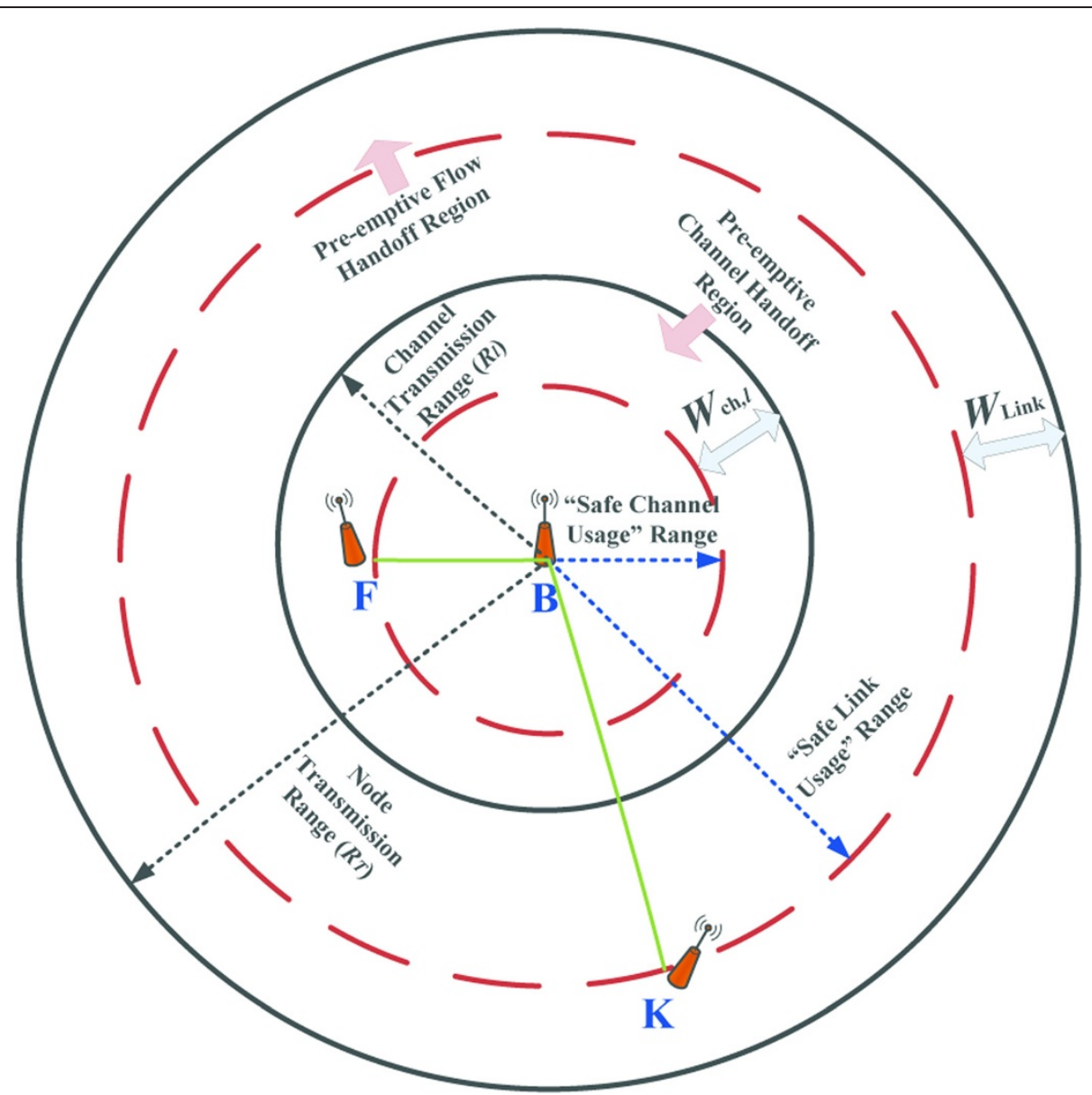

Figure 8 Different preemptive handoff regions.

information is unknown. Using the above proposed channel and local flow handoff prediction scheme with further samples of the transmitted signal; the availability time is estimated without any movement information. Each SU keeps a neighbor signal information table (NSIT). The NSIT involves information about the condition of links with any neighbor. The SU receives and monitors the packets from its neighbors. These packets can be hello packets in the neighbor discovery or data packets in a data transmission. Each node also sends the available channels via hello packets to its neighbors, which are saved in a neighbor channel information table (NCIT). Based on [22], at least three packets are required to estimate the link and channel availability time. Given that at times $T_{1}, T_{2}$, and $T_{3}$, node $\mathrm{F}$ receives the first, second, and third signal with respective powers $P_{1}, P_{2}$, and $P_{3}$ from node $\mathrm{B}$. As Table 2 shows, the SU registers the signal power strength and reception time for each neighbor in the NSIT. When node F receives packets from node $\mathrm{B}$, it updates its NSIT array such that:

$$
P_{3}<P_{2}<P_{1} \text { and } T_{1}<T_{2}<T_{3}
$$

When the distance between two SUs is decreasing, $P_{1}$ will be higher than $P_{2}$ and $P_{3}$. In this case, the power strength $P_{1}$ is set to the latest signal power value, and $P_{2}$ and $P_{3}$ are set based on the new received signal power.

Assume that at time $T$, node $\mathrm{F}$ will receive a signal with power equivalent to the threshold $P_{\mathrm{s}}$, and during time $T_{1}$ to $T$, nodes $\mathrm{A}$ and $\mathrm{B}$ maintain their speeds and directions. Based on [22], the availability time is expressed as follows:

$$
t_{\text {ava }}=\frac{\sqrt{b^{2}-4 a c}-b}{2 a}
$$

Table 2 Neighbor signal information table

\begin{tabular}{lcc}
\hline Power strength/reception time & \\
\hline$P_{3}$ & $P_{2}$ & $P_{1}$ \\
$T_{3}$ & $T_{2}$ & $T_{1}$ \\
\hline
\end{tabular}


here

$$
\begin{aligned}
& \beta=\frac{\left(\sqrt{P_{1} P_{2}} t_{2}+\sqrt{P_{2} P_{3}} t_{3}-\sqrt{P_{1} P_{3}} t_{3}-\sqrt{P_{2} P_{3}} t_{2}\right)}{\left(t_{2} t_{3}^{2}-t_{3} t_{2}^{2}\right) \sqrt{P_{2} P_{3}}}, \\
& a=t_{2} \sqrt{P_{2} P_{s}} \beta, \quad b=\sqrt{P_{s}}\left(\left(\sqrt{P_{1}}-\sqrt{P_{2}}\right) \cdot-t_{2}^{2} \sqrt{P_{2}} \beta\right), \\
& c=t_{2} \sqrt{P_{2} P_{s}}-t_{2} \sqrt{P_{1} P_{2}}, t_{2}=T_{2}-T_{1}, t_{3}=T_{3}-T_{1}, t_{a v i}=T-T_{1}
\end{aligned}
$$

When the $P_{\mathrm{s}}$ is replaced with the $P_{\mathrm{CTR}, l}$, the channel availability time between two communicating nodes for the channel of type $l\left(t_{\mathrm{ava}, T_{l}}\right)$ is an estimated value. The power signal $P_{\mathrm{s}}$ is also replaced by $P_{\mathrm{NTR}}$ to calculate the link availability time $\left(t_{\mathrm{ava}, L}\right)$ between two communicating nodes.

\subsection{Channel allocation scheme}

After a route is established, any node in the route will monitor its next hop to predict the handoff. The entire nodes in the route also perform the spectrum sensing to monitor the PU's activity. Any handoff prediction due to the node mobility or channel quality degradation leads to channel handoff initiation. PU detection in sensing part also initiates the spectrum handoff. When two nodes go far away and there is no more available channel to support their communication, these nodes initiate the local flow handoff, which necessitates the spectrum handoff. In this part, the proposed channel allocation scheme in the decision part is proposed. The proposed scheme is introduced based on the unified formula in (21) for channel availability in CR-MANETs.

We define the $D_{l-1} l$ as the case in which $R_{l-1}<d<R_{l}$, where $d$ is the length of the hop. To give a fair opportunity to the involved SUs in the longer hops, a weight parameter is proposed in the system considering the hop length and the transmission range of the available channels as follows:

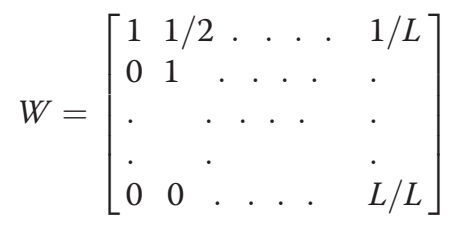

$W$ is the weight matrix with the size of $L \times L$. The row shows the various cases $\left(D_{i i-1}\right)$ based on the hop length, and the column shows the available channel type $\left(T_{l}\right)$. Each element of $W$ indicates the different location weight and is defined as follows:

$$
w_{T_{l}, D_{i-1} i}= \begin{cases}0 & l<i \\ 1 & l=i \\ i / l & l>i\end{cases}
$$

As we have shown in (21), the SU's mobility has a crucial role on the probability of channel availability in
CR-MANETs. Hence, the hop length, which indicates the SU's mobility, is considered for the channel allocation. The parameter $h_{i, l}$ is proposed to show the possibility of using the channel of type $l$, detected by both nodes belonging to the hop $I, H_{i}$. This parameter is defined as follows:

$$
h_{H_{i, T_{l}}}= \begin{cases}1 & \text { for } D_{i-1}, i \leq l \\ 0 & \text { for } D_{i-1}, i>l\end{cases}
$$

As previously mentioned, there is a number of $C_{l}$ channels of type $l$. Each channel of type $l$ is shown by $C_{l z}$ such that $1<z<\mathrm{C}_{l}$. The channel $z$ of type $l$ is used by the nodes belonging to the hop $i$, when it is available for both member nodes. The parameter $\eta$ is proposed to show the possibility of using the channel $z$ of type $l$ for communicating in hop $i$. This parameter is defined as follows:

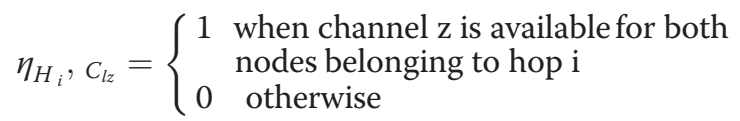

To avoid handing off the data transmission to a channel with a short availability time, the channel availability time must be greater than a threshold. Hence, the parameter $\xi$ is proposed to show the possibility of using the channel of type $l$ for data transmission in hop $i$. The parameter $\xi$ is defined as follows:

$$
\boldsymbol{\xi}_{H_{i}, T_{l}}= \begin{cases}1 & \text { for } t_{\text {ava }, T_{l}, H_{i}} \geq t_{t h} \\ 0 & \text { otherwise }\end{cases}
$$

Finally, the channel allocation metric for channel $z$ of type $l$ is defined as follows:

$$
A M_{C_{l z}}=\left(\xi_{H_{i}, T_{l}}\right) \cdot\left(\eta_{H_{i}}, C_{l z}\right) \cdot\left(h_{H_{i, T_{l}}}\right) \cdot\left(w_{T_{l}, D_{i-1} i}\right) \cdot\left(t_{\text {ava }, T_{l}, H_{i}}\right)
$$

Suppose that the set $C_{H_{i}}$ shows the set of detected channels by two nodes belonging to the hop $i$. This set is composed of channels of different types, and it is shown as follows:

$$
C_{H_{i}}=\cup_{l=1}^{L} C_{H_{i}, l}
$$

The set of available and detected channel used for communication in hop $i$ is defined as follows:

$$
C_{H_{i}, \text { usable }}=\cup_{l=1}^{L}\left[\bigcup_{z \in C_{H_{i, l}}}\left(C_{H_{i, l}} \cdot \boldsymbol{\xi}_{H_{i}, T_{l}} \cdot \eta_{H_{i}}, C_{l z} \cdot h_{H_{i, T_{l}}}\right]\right.
$$

In the decision part, channel allocation scheme chooses the channel $z$ which maximizes the channel allocation metric as follows: 


$$
z^{*}=\arg \max \left\{A M_{C_{l z}}\right\}, \quad z \subseteq C_{H_{i}, \text { usable }}
$$

\subsection{Handoff initiation and connectivity maintenance}

To maintain end-to-end connectivity, topological variations and channel quality degradation due to node mobility are addressed using the handoff request (HREQ) packets. Also, the variations in spectrum availability because of the PU's activity are addressed using the primary user handoff request (PU-HREQ). The single-hop PU-HREQ packet informs the neighbor nodes that the PU's activity has been detected on a special channel and directs them to select another unused channel for data transmission. On the other hand, the HREQ is applied to inform the next hop node that the current link is breaking due to node mobility or channel quality degradation. If channel handoff occurs and no more empty channel is available to maintain the route, local flow handoff, a preemptive approach to address the route breaking, is applied.

In terms of the PU-HREQ, once an SU detects the PU's activity on a special channel, e.g., channel $C_{l i}$, the $\mathrm{SU}$ discards all the entries through channel $C_{l i}$ and informs its neighbors that the channel is busy using a PU-HREQ. The PU-HREQ packet contains the available and detected channels of the current SU. The SUs that receive the PU-HREQ invalidate the entries through $C_{l i}$ that involve the PU-HREQ source. Using the NSIT and NCIT based on the introduced channel allocation scheme, the SU that receives the PU-HREQ finds the channel $z$ that maximizes the channel allocation metric described by (39). When the optimal channel is found, the node sends a handoff reply (PU-HREP) back. This PU-HREP contains the new channel information to perform handoff into it and continue the data transmission.

In terms of topological variation due to node mobility or channel quality degradation, once the SU predicts the handoff, it broadcasts a single-hop channel HREQ packet to its next hop node. When the next node receives the HREQ packet, it makes a decision using the NSIT and NCIT, by a method similar to the procedure for PU-HREQ.

In Figure 9, for example, when node B predicts the entrance of node $\mathrm{F}$ in the preemptive local flow handoff region, node $B$, which uses the link as the next hop, broadcasts a single-hop local flow HREQ (LFHREQ) which contains the next hop node's ID through the common control channel (CCC). Local flow handoff is performed to find an intermediate node for the responsibility of the data transmission between nodes B and F. The selected node is located in the transmission range of both nodes $\mathrm{B}$ and $\mathrm{F}$. A neighbor node that receives the LFHREQ will search its neighbor information table

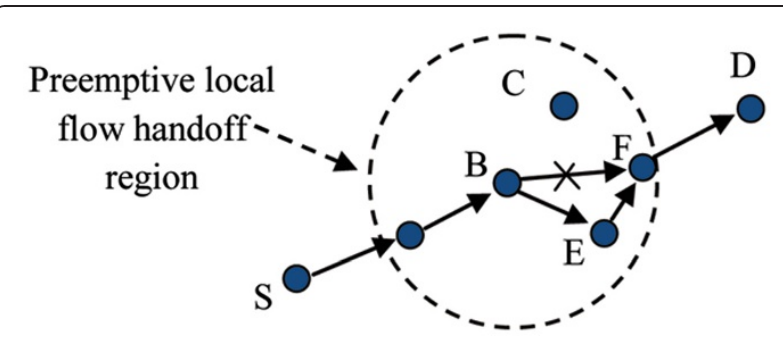

Figure 9 Local flow handoff concept.

(NIT) to determine whether node F is in its NIT or not. If node $\mathrm{F}$ has been registered as a neighbor node in the intermediate node's NIT, the intermediate node estimates the life time connectivity between itself and nodes $\mathrm{B}$ and F. In Figure 9, nodes $\mathrm{E}$ and $\mathrm{C}$ are the proper nodes. Suppose that $t_{\mathrm{ava}, \mathrm{L}, \mathrm{EF}}$ and $t_{\mathrm{ava}, \mathrm{L}, \mathrm{BE}}$ indicate the life time connectivity from $\mathrm{E}$ to $\mathrm{F}$ and from $\mathrm{B}$ to $\mathrm{E}$, respectively. The minimum amount of $t_{\mathrm{ava}, \mathrm{L}, \mathrm{EF}}$ and $t_{\mathrm{ava}, \mathrm{L}, \mathrm{BE}}$ is considered as the life time of the route $\mathrm{B} \rightarrow \mathrm{E} \rightarrow \mathrm{F}$. Then, the intermediate node sends the route life time in a packet as a local flow handoff reply to node $B$.

In case that the route life time is larger than a threshold amount $\left(T_{\mathrm{th}}\right)$, node $\mathrm{E}$ is a candidate for local flow handoff. Node B compares all the life time connectivity information received from its neighbors. Then, it selects the best candidate through which the $\mathrm{B}$ and $\mathrm{F}$ maintain the longest life time.

Suppose that in Figure 9, the node E is the best candidate for the local flow handoff. Node B sends a handoff request $(\mathrm{HR})$ to node $\mathrm{F}$ through node $\mathrm{E}$ using $\mathrm{CCC}$. The HR contains information, such as the ID of node $B$ as the local source, the ID of node F as the local destination, and the channel availability list of the current node. To avoid loops, node E ignores the HR packets when there is corresponding routing information. Once node $\mathrm{E}$ receives the $\mathrm{HR}$ as a candidate for local flow handoff, then:

- Node E compares its own available channels $C_{\text {ava,int }}$ with the available channels of the local source $C_{\text {ava, }}$, locs in HR. The usable channel set in this hop can be shown as follows:

$$
C_{H_{i}, \text { usable }}=\cup_{l=1}^{L}\left[C_{\text {ava,int }, l} \cap C_{\text {ava }, \text { locs }, l} \cdot h_{H_{i, T_{l}}}\right]
$$

- Node E determines the channel $z$, which maximizes the channel allocation metric from the channel set $C_{H_{i} \text {,usable }}$, using (39).

- Let $z$ be the selected channel, node E updates HR with its own information, the ID of node $\mathrm{B}$ as the local source, the ID of node $\mathrm{F}$ as the local destination, and its available channel list. 


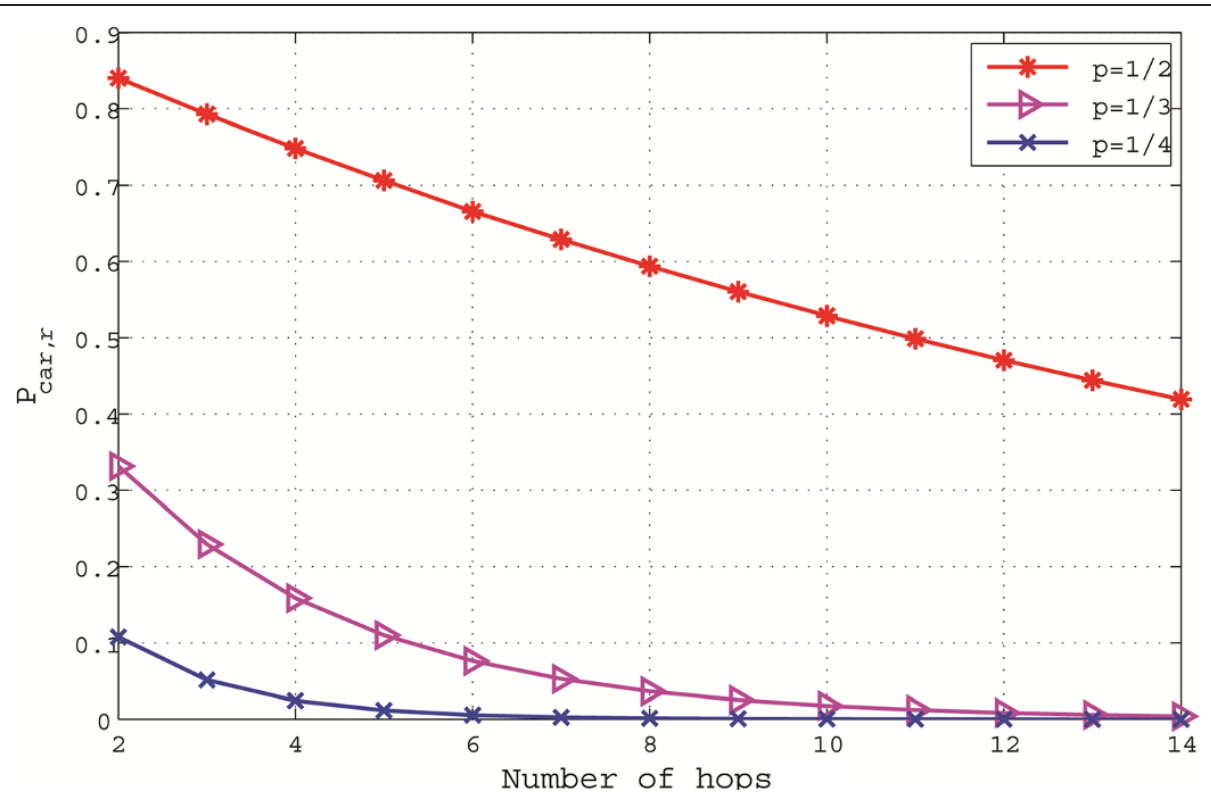

Figure 10 The effect of the activity of the PU on the probability of successful routing with $C=10$.

- Once the CCC is available, it sends the HR to the local destination node F. Finally, this HR will be saved in a handoff table.

When the local destination node $\mathrm{F}$ receives the HR, like intermediate node $\mathrm{E}$, it selects a proper channel for its upper hop. The local destination node $\mathrm{F}$ sends the handoff acknowledgment (HA) packet back to the local source node $B$ through node $E$. The HA message sets up a new route between $B$ and $F$, which is the route $\mathrm{B} \rightarrow \mathrm{E} \rightarrow \mathrm{F}$. Then, the routing tables in nodes $\mathrm{B}, \mathrm{E}$, and $\mathrm{F}$ are updated. Once the new route is established, the data flow will be passed along the new route. In the case that the local flow handoff is not possible, the global flow handoff will be performed by the source node.

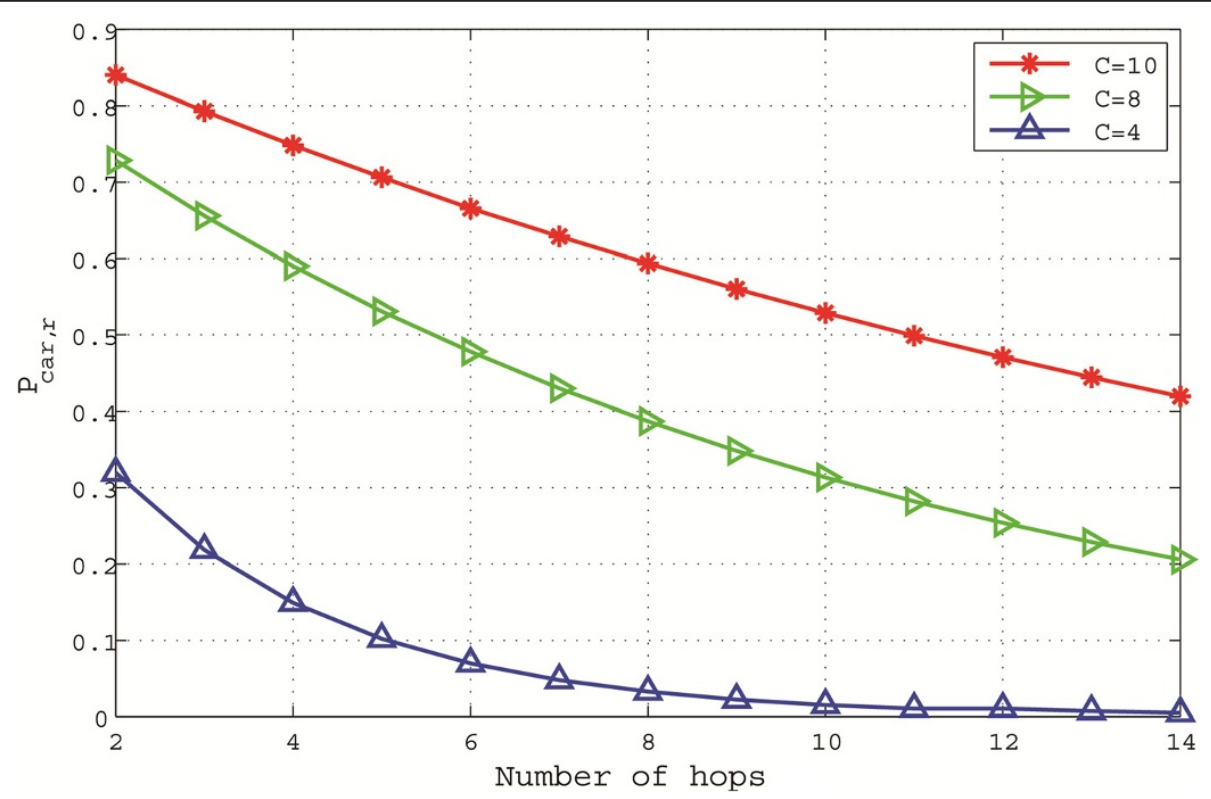

Figure 11 The effect of the number of available channels on the probability of successful routing with $p=1 / 2$. 


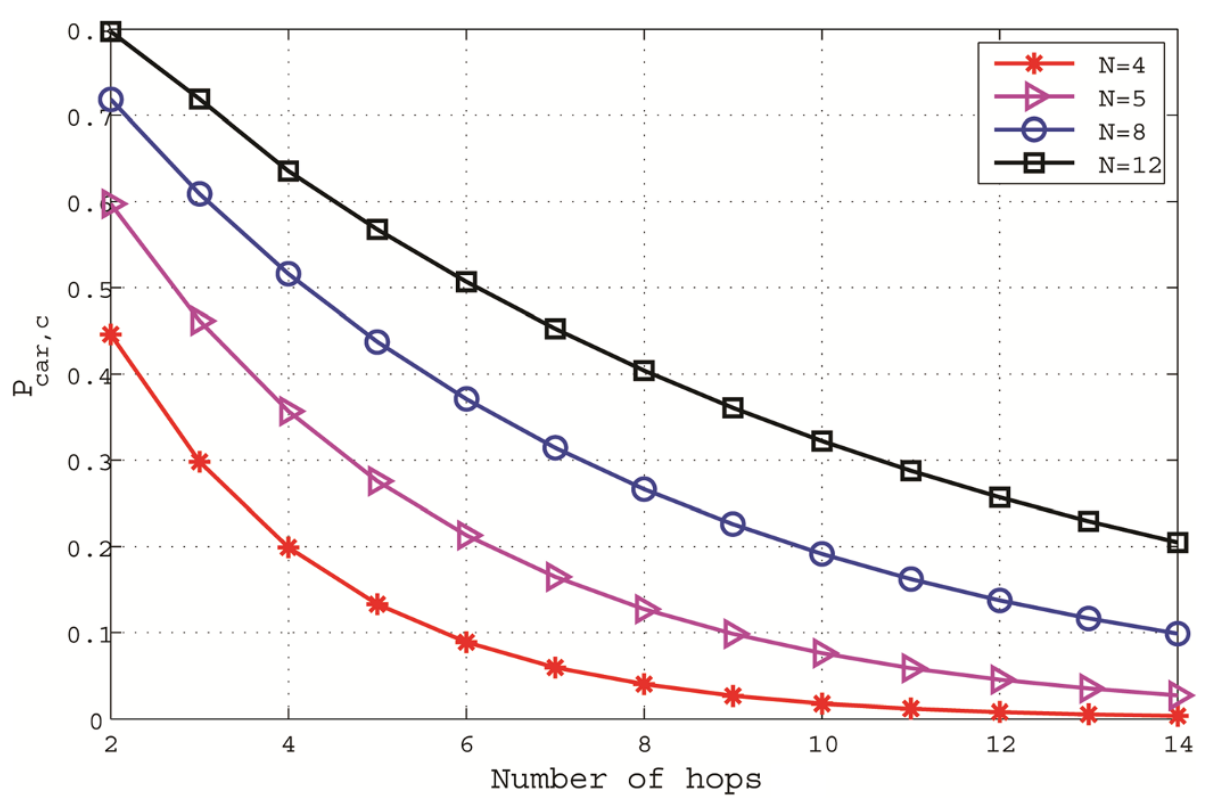

Figure 12 The effect of $\lambda$ and $N$ on $P_{\text {car,c }}$.

\section{Results and discussion}

\subsection{Probability of channel availability in CR-MANETs}

In the first part, the probability of channel availability in CR-MANETs is investigated considering the effects of different parameters such as the number of SUs, node and channel transmission range, and the number of available channels in the network. Figures 10 and $11 \mathrm{ex}$ press the effect of the activity of the PU and the number of available channels on the probability of successful rerouting of the $\mathrm{SU}$, respectively. Figure 10 plots the probability of successful rerouting based on the number of hops in the route and the constant number of homogeneous channels $C=10$. Meanwhile, Figure 11 shows the effect of the number of available homogeneous channels on the probability of successful rerouting with $p=1 / 2$.

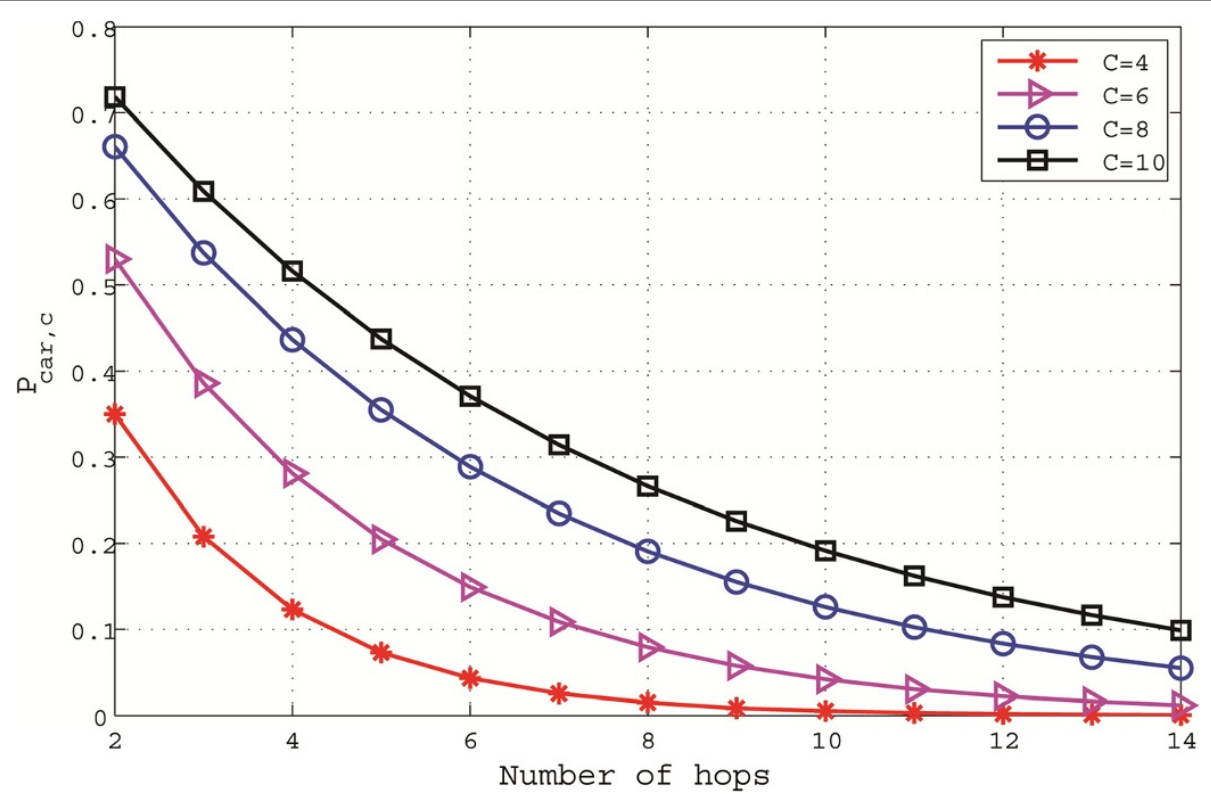

Figure 13 The effect of number of available channels on $P_{\text {car, } c}$. 


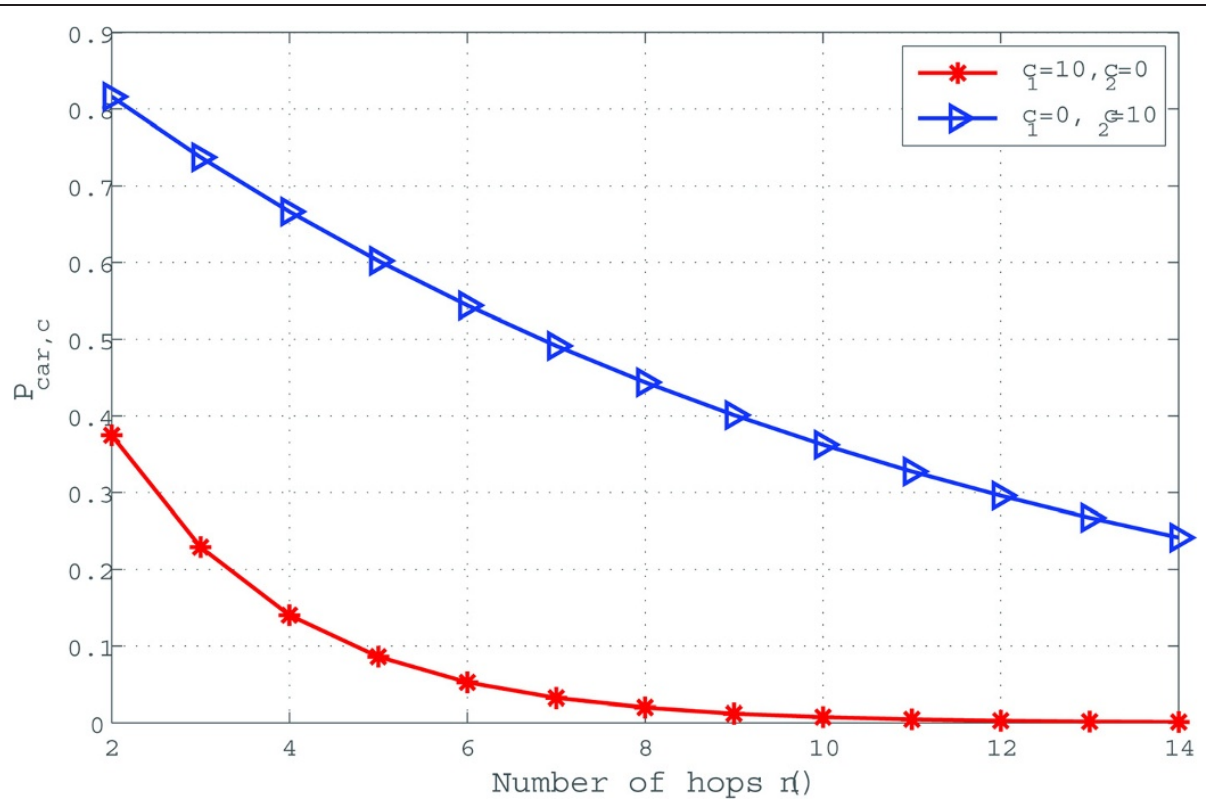

Figure 14 The effect of channel transmission range on the $P_{\text {car, }}$.

Based on these figures, we can see that the probability of channel availability through a route is dependent on the activity of the PU and the total number of available channels, and it decreases as the number of hops increases.

7.2 The effects of different parameters on the $P_{\text {car, }}$ In the second part, we show the effect of different parameters on the $P_{\mathrm{car}, c}$. We suppose that there are two types of channels with a transmission range of $R_{1}=75 \mathrm{~m}$ and $R_{2}=125 \mathrm{~m}$ and a maximum node transmission range of $R_{\mathrm{T}}=150 \mathrm{~m}$. We also assume that the activity of the PU on different channels is identical.

Figure 12 shows the effect of the node density on the $P_{\text {car, } c}$. Here, the number of channels is constant $C=10$, $c_{1}=5, c_{2}=5$. This figure shows that $P_{\mathrm{car}, c}$ has an increasing trend when $\lambda$ increases. Figure 13 presents the effect of the number of available channels on $P_{\text {car, }}$ under a constant PU activity of $p=1 / 2$ and $N=8$. This figure also shows a decreasing trend with an increasing number of hops. It also shows the high dependency between the number of available channels and $P_{\text {car, } c}$.

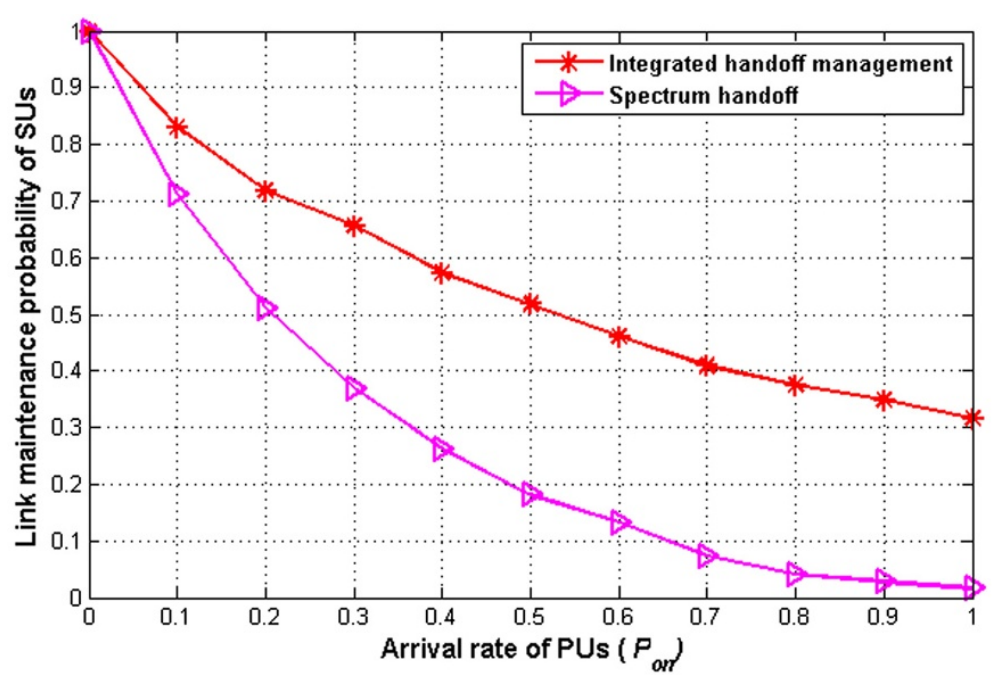

Figure 15 Route maintenance probability for the SUs vs. arrival rate of primary users $\left(P_{\text {on }}\right)$. 


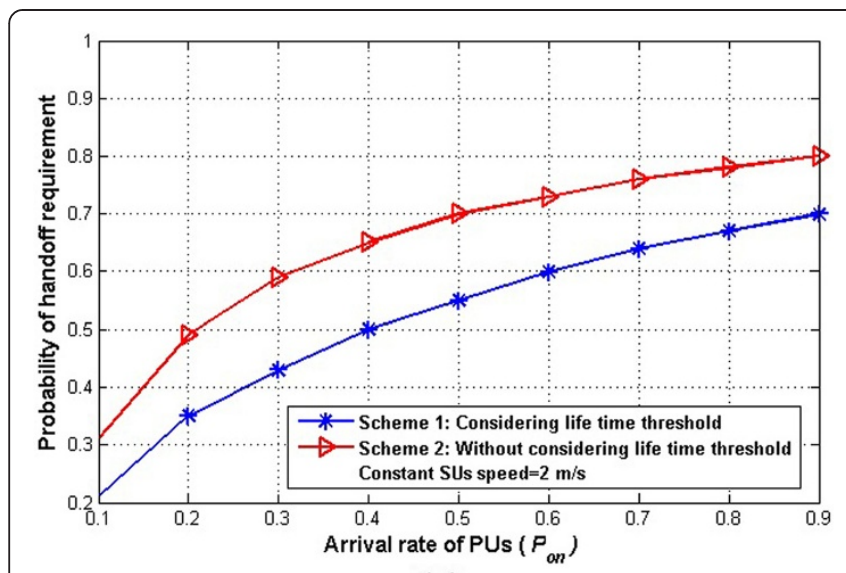

(a)

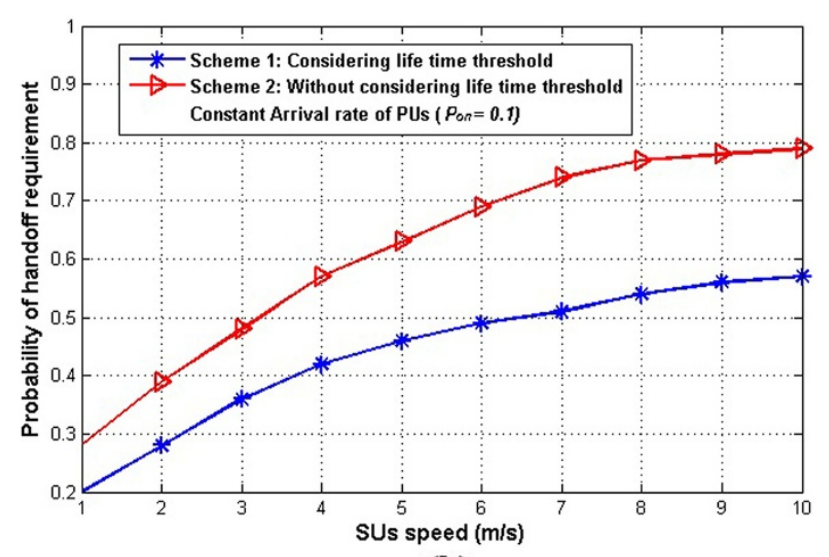

(b)

Figure 16 The expected amount of handoff requirements for the SUs vs. PU's arrival rate (a) and SU's velocity (b).

Figure 14 shows the effect of channels' type on the $P_{\text {car }, c}$. In this figures, $p=1 / 2, C=10$, and $N=8$. The result implies that the $P_{\mathrm{car}, c}$ is dependent on the channel transmission range.

\subsection{Link maintenance, handoff blocking, and spectrum handoff investigation}

In this section, performance comparisons of different schemes are conducted using network simulator 2 (NS-2) [23]. The SU's route maintenance probability, handoff blocking probability, and the number of anticipated spectrum handoff are investigated. There is a total number of available channels $C=10$, classified into two different types, $c_{1}=5$ and $c_{2}=5$. The transmission ranges of different channel types and the node transmission range are set to $R_{1}=75 \mathrm{~m}, R_{2}=125 \mathrm{~m}$, and $R_{\mathrm{T}}=150 \mathrm{~m}$, respectively. The mobile $\mathrm{SUs}$ are distributed in a network with $3,000 \mathrm{~m} \times 3,000 \mathrm{~m}$ area, and

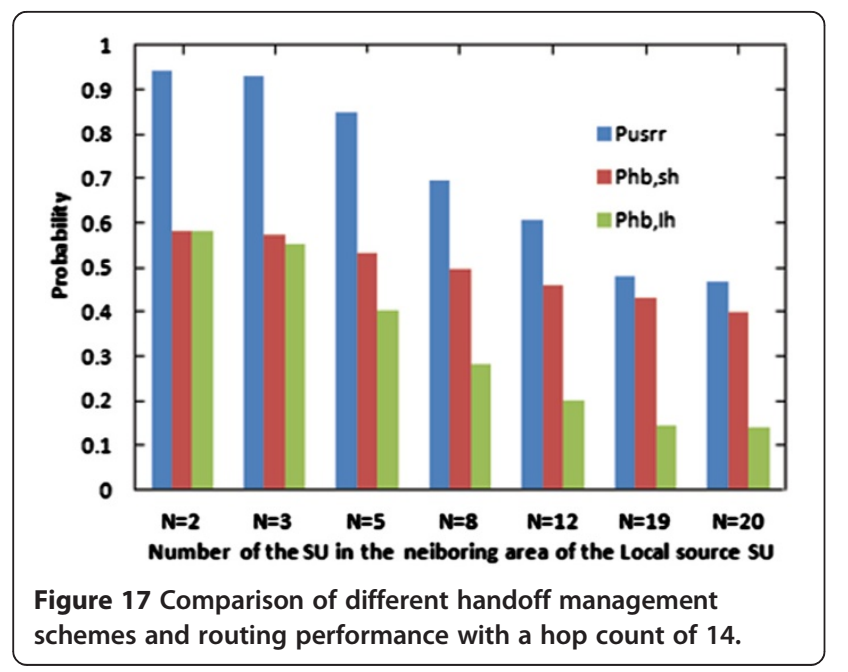

their speed is uniformly distributed from 1 to $10 \mathrm{~m} / \mathrm{s}$. Both channel usage time and the link life time threshold are set to $8 \mathrm{~s}$.

In order to study the route maintenance probability, two different handoff management schemes are considered. The first scheme only deploys the spectrum handoff, while the second scheme deploys the PIHM in which the local flow handoff will be added to the management system rather than the spectrum handoff. Both two different schemes consider the handoff threshold for the preemptive handoff region, life time threshold, and position weight. Figure 15 verifies the effect of local flow handoff on the route maintenance probability. It is expressed that the proposed integrated handoff approach efficiently improves the route maintenance probability. In this approach, the data flow is transferred to the nearby users to keep the communication, while the first scheme only uses the spectrum handoff to keep the

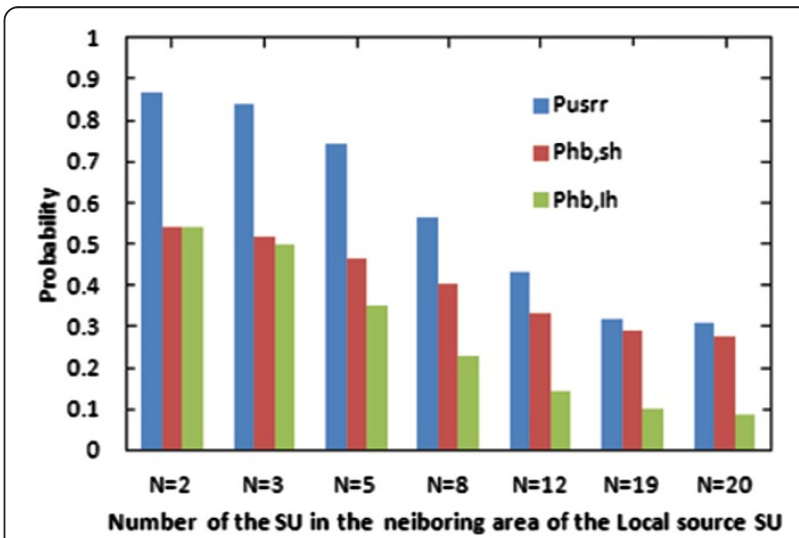

Figure 18 Comparison of different handoff management schemes and routing performance with an expected hop count of 5 . 
route. In case that there is no proper node, the handoff blocking occurs.

Figure 16 shows the expected amount of handoff requirement for the SUs. To evaluate the effects of the PU's activity and the SU's mobility, the PU's arrival rate and SU's velocity vary in the Figure 16 . From this figure, it is stated that using the life time threshold in the channel allocation procedure decreases the number of required handoff. Based on this figure, the SU's mobility also affects the link availability time, and it has an important effect on the performance of the CR network. Hence, the mobility of SUs is considered in the handoff management schemes.

The probability of unsuccessful route maintenance is defined as the probability of spectrum handoff blocking $\left(P_{\mathrm{hb}}\right)$. Figures 17 and 18 indicate that the spectrum handoff blocking probability in PIHM scheme $\left(P_{\mathrm{hb}, \mathrm{Ih}}\right)$ is lower than the probability of spectrum handoff blocking in scheme deploying only spectrum handoff $\left(P_{\mathrm{hb}, \mathrm{sh}}\right)$. This is due to deploying the local flow handoff in the integrated management scheme. On the other hand, the $P_{\mathrm{hb}, \mathrm{Ih}}$ is significantly lower than the probability of unsuccessful rerouting $\left(P_{\text {usrr }}\right)$. Hence, deploying the local flow handoff can be more efficient than the global routing. These figures also indicate, once the number of SUs in the network increases, the probability of handoff blocking decreases, since the probability of finding the proper nodes to perform local flow handoff increases. It is stated that the effect of the number of SU's on the $P_{\mathrm{hb}, \mathrm{Ih}}$ and $P_{\mathrm{usrr}}$ is higher than the effect of the number of SU's on the $P_{\mathrm{hb}, \mathrm{sh}}$. Comparing Figures 17 and 18, it can be concluded that the $P_{\mathrm{hb}}$ decreases when the number of hops decreases.

\section{Conclusions}

Spectrum handoff is extremely challenging in cognitive radio networks, especially in CR-MANETs because of frequent topological variations, limited power and channel transmission range, bandwidth constraints, and lack of a central controlling entity. In this paper, channel switching in CR-MANETs, for an established route, is modeled, and the probability of channel availability in this route is calculated based on PU's activity, SU's mobility, and channel heterogeneity. The study reveals that the channel heterogeneity and the SU's mobility must be considered as important factors, which affect the performance of handoff management in the CRMANETs. Based on the introduced scenarios for handoff initiation in CR-MANETS, a proactive integrated handoff management (PIHM) is introduced for this network. The results show that the proposed integrated handoff management scheme achieves more data transmission opportunities. Based on the results, the route maintenance probability is increased, while the number of spectrum handoff is reduced.

\section{Competing interests}

The authors declare that they have no competing interests.

\section{Acknowledgments}

This work was supported in part by the Ministry of Science, Technology and Innovation (MOSTI) Malaysia, and Research Management Center (RMC), University Technology Malaysia under GUP research grant no. R. J130000.7923.4S063.

\section{Author details}

${ }^{1}$ Faculty of Electrical Engineering, University Technology Malaysia, Skudai 81300, Malaysia. ${ }^{2}$ Electrical Engineering Department, University of North Dakota, Grand Forks, ND 58202, USA.

Received: 17 December 2012 Accepted: 19 August 2013

Published: 5 September 2013

\section{References}

1. FCC, Notice of proposed rulemaking and order, no.03-222 (Federal Communications Commission, Washington, DC, 2003)

2. Y Ding, L Xiao, Channel allocation in multi-channel wireless mesh networks. Comput. Commun. 34, 803-815 (2011)

3. N Guoqin, J Duan, J Su, D Qiu, Spectrum sharing based on spectrum heterogeneity and multi hop handoff in centralized cognitive radio networks, in 20th Annual WOCC (Newark, NJ, 2011), p. 1

4. L Giupponi, Al Perez-Neira, Fuzzy-based Spectrum Handoff in Cognitive Radio Networks, in 3rd International Conference on Cognitive Radio Oriented Wireless Networks and Communications CrownCom (Singapore, 2008), pp. 1-6

5. W Feng, J Cao, C Zhang, C Liu, Joint Optimization of Spectrum Handoff Scheduling and Routing in Multi-hop Multi-radio Cognitive Networks, in 29th IEEE International Conference on Distributed Computing Systems (Montreal, QC, 2009), pp. 85-92

6. Y Song, J Xie, ProSpect: a proactive spectrum handoff framework for cognitive radio ad hoc networks without common control channel, IEEE trans. Mobile Comput. 11(99), 1 (2011)

7. Y Song, J Xie, Performance analysis of spectrum handoff for cognitive radio ad hoc networks without common control channel under homogeneous primary traffic. IEEE INFOCOM 2011(10-15), 3011-3019 (2011)

8. Z Damljanovic, Mobility management strategies in heterogeneous cognitive radio networks. Springer Sci. Mag. 18(1), 4-22 (2010)

9. J Duan, Y Li, An optimal spectrum handoff scheme for cognitive radio mobile ad hoc networks. AECE 11, 11-16 (2011)

10. K Wu, J Harms, Performance study of proactive flow handoff for mobile ad hoc networks. Wireless Netw. Springer 12, 119-135 (2006)

11. P Abhilash, S Perur, S Lyer, H Router, An approach for preemptive route repair in mobile ad hoc networks. Lect. Notes Comp. Sci. 2552, 347-357 (2002)

12. M Caleffi, IF Akyildiz, L Paura, OPERA, optimal routing metric for cognitive radio ad hoc networks. IEEE Trans. Wireless Commun. 11(8), 2884-2894 (2012)

13. A Chehata, W Ajib, H Elbiaze, An on-demand routing protocol for multi-hop multi-radio multi-channel cognitive radio networks, in Wireless Days 2011 IEEE conferences (Niagara Falls, ON, 2011), pp. 1-5

14. AS Cacciapuoti, M Caleffi, L Paura, Reactive routing for mobile cognitive radio ad hoc networks. Ad. hoc. networks 10(5), 803-815 (2011)

15. S Nejatian, SK Syed Yousof, NM Abdol Lattif, V Asadpour, Proactive Integrated handoff management in CR-MANETs: a conceptual model, in IEEE Symposium on Wireless Technology and Applications (ISWTA) (Bandung, 2011), pp. 33-38

16. WY Lee, IF Akyildiz, Spectrum-aware mobility management in cognitive radio cellular networks. IEEE Trans Mobile Comput. 11(4), 529-542 (2012)

17. PE Pfeiffer, Concepts of probability theory (Courier Dover Publications, Mineola, NY, 1978), pp. 47-48. ISBN 978-0-486-63677-1

18. YC Cheng, TG Robertazi, Critical connectivity phenomena in multi hop radio models. IEEE Trans. Communications 37(7), 770-777 (1989)

19. T-C Hou, VO-K Li, Transmission range control in multihop packet radio networks. IEEE Trans. Commun. 32(1), 38-44 (1986)

20. T. Goff, N.B. Abu-Ghazleh, D.S. Phatak, R. Kahvecioglu, Preemptive Routing in Ad Hoc Networks, in Proceedings of ACM SIGMOBILE (Rome, 2001), pp. 43-52 
21. QS Guan, FR Yu, SM Jiang, Prediction-based topology control and routing in cognitive radio mobile ad hoc networks, in Proceedings of IEEE INFOCOM (San Diego, CA, 2010), pp. 1-6

22. L Qin, T Kunz, Increasing Packet Delivery Ratio in DSR by Link Prediction, in Proceedings of the 36th Hawaii IEEE International Conference on System Sciences (HICSS'03) (Big Island, 2003), pp. 6-9

23. UC Berkeley, LBL, USC/ISI, Xerox PARC, The Vint Project, the ns Manual (formerly ns Notes and documentation), in ed. by K. Fall, K. Varadhan (UC Berkeley, Berkeley, 2010)

doi:10.1186/1687-1499-2013-224

Cite this article as: Nejatian et al.: Proactive integrated handoff

management in cognitive radio mobile ad hoc networks. EURASIP Journal on Wireless Communications and Networking 2013 2013:224.

\section{Submit your manuscript to a SpringerOpen ${ }^{\circ}$} journal and benefit from:

- Convenient online submission

- Rigorous peer review

- Immediate publication on acceptance

- Open access: articles freely available online

- High visibility within the field

- Retaining the copyright to your article 